\title{
Gravity Modeling Effects on Surface-Interacting Vehicles in Supersonic Flight
}

\author{
Michael M. Madden* \\ NASA, Hampton, VA, 23681
}

\begin{abstract}
A vehicle simulation is "surface-interacting" if the state of the vehicle (position, velocity, and acceleration) relative to the surface is important. Surface-interacting simulations perform ascent, entry, descent, landing, surface travel, or atmospheric flight. The dynamics of surface-interacting simulations are influenced by the modeling of gravity. Gravity is the sum of gravitation and the centrifugal acceleration due to the world's rotation. Both components are functions of position relative to the world's center and that position for a given set of geodetic coordinates (latitude, longitude, and altitude) depends on the world model (world shape and dynamics). Thus, gravity fidelity depends on the fidelities of the gravitation model and the world model and on the interaction of these two models. A surfaceinteracting simulation cannot treat gravitation separately from the world model. This paper examines the actual performance of different pairs of world and gravitation models (or direct gravity models) on the travel of a supersonic aircraft in level flight under various starting conditions.
\end{abstract}

\section{Nomenclature and Acronyms}

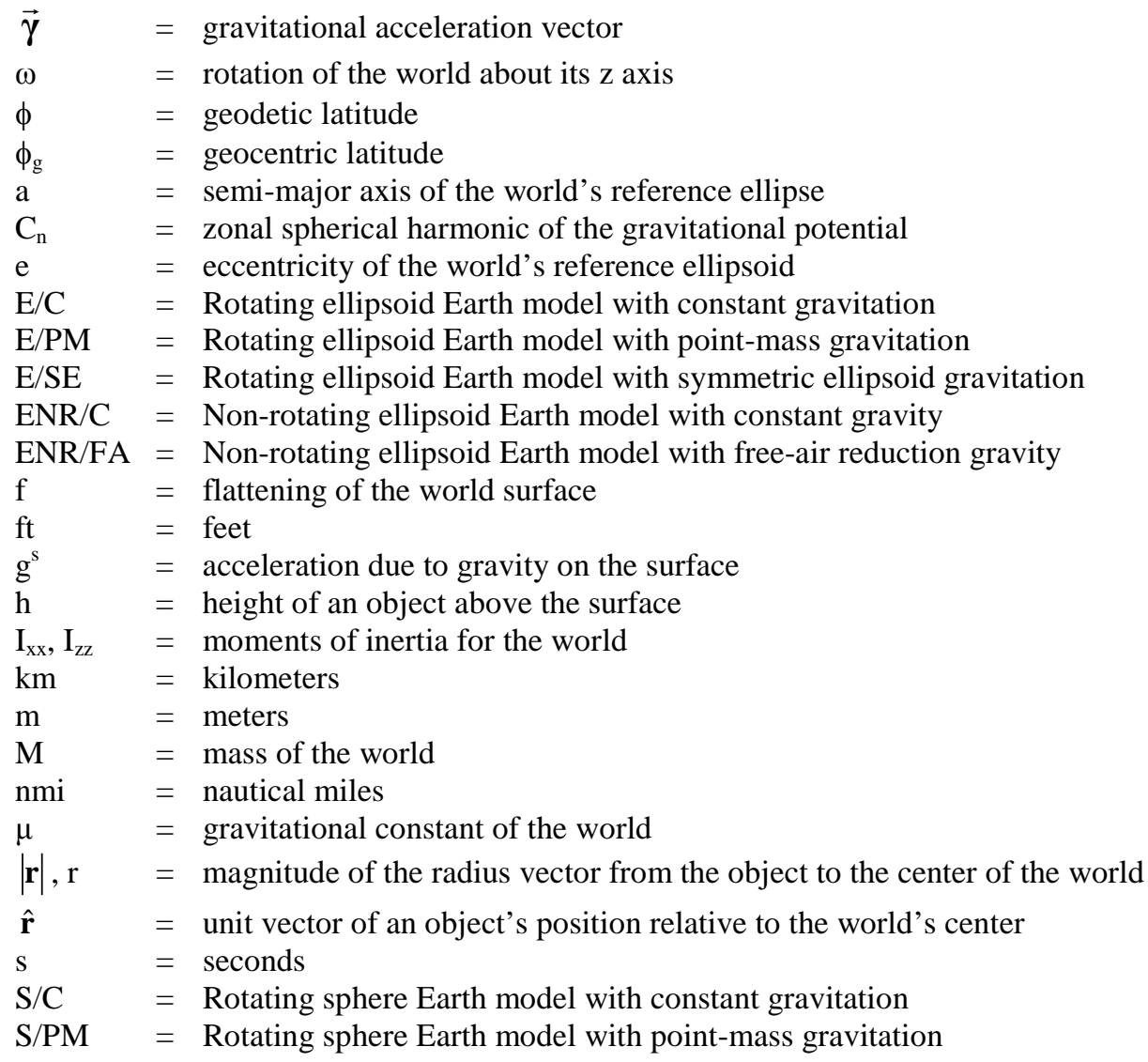

${ }^{*}$ Chief Scientist, Simulation Development and Analysis Branch, Mail Stop 125B, Senior Member AIAA. 
$\mathrm{S} / \mathrm{SE}=$ Rotating sphere Earth model with symmetric ellipsoid gravitation

$\mathrm{SNR} / \mathrm{C}=$ Non-rotating sphere Earth model with constant gravity

SNR/FA = Non-rotating sphere Earth model with free-air reduction gravity

WGS84 = World Geodetic System 1984

$\mathrm{x}, \mathrm{y}, \mathrm{z}=$ Earth-fixed Cartesian coordinates

\section{Introduction}

erospace simulations can model worlds, such as the Earth, with differing levels of fidelity. The simulation may Arepresent the world as a plane, a sphere, an ellipsoid, or a high-order closed surface. The world model may or may not reproduce the rotation of the world in space. Likewise, the simulation can model gravity or the gravitation component, as appropriate, using models of differing fidelities; examples include constant gravity, constant gravitation, free-air reduction, point-mass gravitation, and a spherical-harmonic expansion of the geopotential. The user may select lower fidelity models based on computational limits, a need for simplified analysis, or comparison to other data. Simulation products can model the world and gravity or gravitation independently, allowing users to select any combination of world and gravity/gravitation models. For example, the Langley Standard Real-Time Simulation in C++ (LASRS++ $)^{1}$ presents world and gravity/gravitation models as independent user selections. Independently selecting the gravity/gravitation model and the world model does not necessarily result in a combined fidelity equivalent to the fidelity of the individual models. Some pairs produce degraded fidelity. ${ }^{2}$

This study examines the actual performance of paired world and gravity or gravitation models ("world-gravity pairs") in a simulation using the Earth. The choice of world-gravity pairs is based on prior analytical work in Reference 2; the pairs are described in section II.C. The selected world-gravity pairs are applied to a simulation of a supersonic aircraft in level flight that is initialized to various locations and track angles. Performance of the worldgravity pairs is measured as the deviation of the aircraft's trajectory from the trajectory produced by a reference world-gravity pair. The reference world-gravity pair models a rotating WGS- $84^{3}$ ellipsoid of the Earth whose density is symmetric about the polar axis and the equator. The nine simulation scenarios examined here expand the work in References 4 and 5, which presented the performance of the same world-gravity pairs using a subsonic aircraft simulation. The scenarios are described in section III; the resulting aircraft trajectories are discussed in section IV. The next section provides a brief overview of the world and gravity/gravitation models; more detailed treatment of these models is found in Reference 2.

\section{The Models}

\section{A. World Models}

World models simulate the motion of the world and provide an approximation of its shape. For the purposes of this investigation, a world is a body whose shape is roughly spherical under the influence of its self-gravity. This study examines the sphere and ellipsoid shape models that are commonly used to model the Earth. This study does not address modeling of the Earth as a plane, i.e. the "flat Earth" model. The modeling of gravity or gravitation becomes important only as one travels large distances over the Earth, and "flat Earth" models are valid only under comparatively small distances. To model the Earth as an ellipsoid, this investigation uses the ellipsoid of the World Geodetic System 1984 (WGS84). ${ }^{3}$ To model the Earth as a sphere, this study uses the radius of a sphere with equal surface area to the WGS84 ellipsoid because it minimizes error in the mean surface gravity. ${ }^{2}$

Vehicles in surface interacting simulations are bound to the world by gravitation. Thus, only the world's motion relative to its center of mass, i.e. its rotation, is relevant to surface-interacting dynamics. The Earth has a dominant rotation about the z-axis in Earth-fixed, Earth-centered Cartesian coordinate system. In this study, the Earth's rotation is modeled as a constant $7.292115 \times 10^{-5} \mathrm{rad} / \mathrm{sec}^{3}$. Rotation can be added to the sphere and ellipsoid to increase the overall fidelity of those models or it can be ignored for simplicity. Disabling rotation removes the centrifugal acceleration and Coriolis acceleration terms in the acceleration equation for a vehicle when observed in an Earthfixed frame. The centrifugal acceleration term is a function of vehicle position. Its mean value on the surface of the Earth is $0.23 \%$ of gravity. The Coriolis acceleration term is proportional to the object's velocity. In this investigation, the magnitude of the Coriolis acceleration term is limited to $0.61 \%$ of mean gravity.

Rotation also directly impacts how gravity is modeled. Gravity is the combination of gravitation and centrifugal acceleration. ${ }^{6}$ If rotation is turned off, centrifugal acceleration is not modeled, and a surface interacting simulation must model gravity directly in order to reproduce the free fall of objects as observed from the Earth surface. Modeling gravitation without modeling rotation will produce slightly larger free fall at locations away from the poles. 
(The centrifugal acceleration is zero at the poles). If rotation is modeled, then the simulation should not use a gravity model; doing so will exaggerate the affect of the centrifugal acceleration. Only gravitation models should be selected when rotation is modeled.

\section{B. Gravity and Gravitation Models}

This study examines two gravity models and three gravitation models. The gravity models are constant gravity and the free air reduction. The gravitation models are constant gravitation, point-mass gravitation, and gravitation of a symmetric ellipsoid.

\section{Constant Gravity and Gravitation}

The constant gravity and gravitation models use one value for gravity or gravitation regardless of the vehicle's location. Gravity acts along the surface normal and gravitation acts along the geocentric radius between the vehicle and the center of the Earth. ${ }^{\dagger}$ The gravity constant selected for this model is standard gravity, $9.80665 \mathrm{~m} / \mathrm{s}^{2}$ (32.174 $\left.\mathrm{ft} / \mathrm{s}^{2}\right){ }^{\ddagger}$ The gravitation constant selected for this model is the mean gravitation, $9.82023 \mathrm{~m} / \mathrm{s}^{2}\left(32.219 \mathrm{ft} / \mathrm{s}^{2}\right)$.

\section{Free Air Reduction}

The free air reduction linearly approximates the vertical gradient of gravity in the neighborhood of the reference geoid; the vertical gradient of gravity is approximately $3.086 \times 10^{-6} \mathrm{~s}^{-2} .7$ The free air reduction equation is:

$$
g=g^{s}+3.086 \times 10^{-6} h
$$

where $\mathrm{g}^{\mathrm{s}}$ is the reference gravity on the surface and $\mathrm{h}$ is the altitude. This study uses standard gravity for $\mathrm{g}^{\mathrm{s}}$.

\section{Point-Mass Gravitation}

The point-mass gravitation model applies Newton's universal law of gravitation to a spherically symmetric mass. A spherically symmetric mass can be treated as if all of its mass where located at its center, i.e. a point mass. Equation 2 is the formula for point-mass gravitation:

$$
\vec{\gamma}=-\frac{\mu}{|\mathbf{r}|^{2}} \hat{\mathbf{r}}
$$

\section{Symmetric Ellipsoid Gravitation}

The symmetric ellipsoid gravitation model uses a simplified spherical harmonic expansion that assumes the Earth is an ellipsoid whose mass is symmetric about the pole and equator. The gravitation vector is the gradient of that harmonic expansion. Equations 3 and 4 express the resulting gravitation equations to a degree of four:

$$
\begin{aligned}
& \gamma_{r}=-\frac{\mu}{r^{2}}\left[1+\frac{3}{2}\left(\frac{a}{r}\right)^{2} C_{2}\left(3 \sin ^{2} \phi_{g}-1\right)+\frac{5}{8}\left(\frac{a}{r}\right)^{4} C_{4}\left(35 \sin ^{4} \phi_{g}-30 \sin ^{2} \phi_{g}+3\right)\right] \\
& \gamma_{\phi_{g}}=\cos \phi_{g} \sin \phi_{g} \frac{\mu}{r^{2}}\left(\frac{a}{r}\right)^{2}\left[3 C_{2}+\frac{1}{2}\left(\frac{a}{r}\right)^{2} C_{4}\left(35 \sin ^{2} \phi_{g}-15\right)\right]
\end{aligned}
$$

where,
$\gamma_{r}, \gamma_{\phi_{g}}$
are the components of gravity along the geocentric radius and latitudinal (geocentric) directions
$\phi_{\mathrm{g}} \quad$ is the geocentric latitude ${ }^{\S}$
$\mathrm{r} \quad$ is the geocentric radius
$\mu \quad$ is the universal gravitational constant
a $\quad$ is the semi-major axis of the reference ellipsoid
$\mathrm{C}_{\mathrm{n}} \quad$ are the even zonal harmonic coefficients of degree $\mathrm{n}$

The simulation in this study uses equations expanded to degree eight because the resulting equations match WGS84 published gravitation values to ten significant digits. ${ }^{2}$ For a symmetric ellipsoid mass, the even zonal harmonic

${ }^{\dagger}$ Constant gravitation better predicts surface gravity by a small fraction when applied to the geodetic unit vector. ${ }^{2}$ However, constant gravitation produces lower trajectory errors when applied to the geocentric unit vector. ${ }^{4}$

₹ Standard gravity is a standard unit of acceleration ("g”) and not a physical property of the Earth. However, its error profile against the WGS84 gravity formula shows improved fidelity over the mean gravity which is a physical property. $^{2}$

${ }^{\S}$ Geocentric latitude is the angle between the equatorial plane and the radius from the center of the world to a point. ${ }^{3}$ 
coefficients ( $C_{n}$ where $n$ is even) are functions of the world's mass $(M)$, equatorial radius (a), and moments of inertia (Ixx, Izz) as shown in Equation 5: ${ }^{7,8}$

$$
C_{2}=\frac{I_{z z}-I_{x x}}{M a^{2}} \quad, \quad C_{2 \times n}=(-1)^{n} \frac{3 e^{2 \times n}}{(2 \times n+1)(2 \times n+3)}\left(1-n-5 \times n \frac{C_{2}}{e^{2}}\right)
$$

where $e$ is the eccentricity of the Earth. The simulation used the WGS84 value of $C_{2},-0.00108262982131$ (without normalization). ${ }^{3}$

The symmetric ellipsoid gravitation model includes physical properties of the world model (e.g. eccentricity). Because the sphere's physical properties would reduce the symmetric ellipsoid gravitation model to a point-mass model, the physical properties of the WGS84 reference ellipsoid are substituted when pairing this gravitation model with the sphere model of the Earth. Moreover, the gravitation vector is computed as if the geodetic coordinates of the vehicle on the sphere are at the same geocentric location as those geodetic coordinates on the ellipsoid. As a result, the formula for the symmetric ellipsoid gravitation model is identical between the sphere and ellipsoid world models for a given geodetic coordinate. Reference 5 demonstrated that this approach to applying the symmetric ellipsoid model to a sphere produces the lowest position error, and that the position error is largely due to the differences in surface modeling. The difference in gravity modeling is negligible.

\section{Paring World and Gravity/Gravitation Models}

The world and gravity/gravitation models can be combined to produce ten pairs. This is less than the number of possible permutations because some permutations are eliminated due to incompatible physics. Gravity models are paired only with non-rotating world models. Likewise, gravitation models are paired only with rotating world models. The remaining ten pairs are:

- Rotating ellipsoid Earth with symmetric ellipsoid gravitation (E/SE)

- Rotating ellipsoid Earth with point-mass gravitation (E/PM)

- Rotating ellipsoid Earth with constant gravitation (E/C)

- Rotating sphere Earth with symmetric ellipsoid gravitation (S/SE)

- Rotating sphere Earth with point mass gravitation (S/PM)

- Rotating sphere Earth with constant gravitation (S/C)

- Non-rotating ellipsoid Earth with free air reduction (ENR/FA)

- Non-rotating ellipsoid Earth with constant gravity (ENR/C)

- Non-rotating sphere Earth with free air reduction (SNR/FA)

- Non-rotating sphere Earth with constant gravity (SNR/C)

Because all pairs produce gravity to a surface observer, the pairs will be called world-gravity pairs. The first pair (E/SE) matches the theoretical surface gravity of a rotating Earth in hydrostatic equilibrium and is used as the reference pair against which the trajectory performance of the other pairs is compared. This paper uses the following terms to refer to related groups of gravity models:

- reduced-fidelity, world-gravity pairs = E/PM, E/C, S/SE, S/PM, S/C, ENR/FA, ENR/C, SNR/FA, and SNR/C

- rotating world-gravity pairs = E/PM, E/C, S/SE, S/PM, and S/C (excludes E/SE unless otherwise noted)

- non-rotating world-gravity pairs = ENR/FA, ENR/C, SNR/FA, and SNR/C

- ellipsoid world-gravity pairs = E/PM, E/C, ENR/FA, and ENR/C (excludes E/SE unless otherwise noted)

- sphere world-gravity pairs = S/SE, S/PM, S/C, SNR/FA, and SNR/C

\section{Method and Prior Work}

This study uses nine routes to evaluate the surface-relative dynamics of a supersonic aircraft under each worldgravity pair. The aircraft flies without an active control system ("open-loop”) to freely react to changes in gravity; the aircraft also does not model fuel burn to maintain thrust during the entire flight. Table 1 presents the initial conditions for each route. These routes are derived from the routes in References 4 and 5 except that the initial altitude is $40,000 \mathrm{ft}$ (a 16,000 ft increase) and the initial speed is $1355 \mathrm{ft} / \mathrm{s}$ (doubling the speed). The initial speed corresponds to a mach number of 1.4. The initial speed and altitude remain the same for each route. The aircraft is initialized to straight and level flight, solving for zero accelerations along the body $\mathrm{x}$ and $\mathrm{z}$ axis. Initial orientation differs only in pitch to balance lift against the force of gravity. Initial angular velocity differs to maintain orientation as the aircraft flies over the curved surface of the Earth. The initial elevator deflection differs to zero pitch accelerations. The simulation elapsed time is set to 12 hours during which the aircraft flies approximately 9600 nautical miles. The simulation integrates the vehicle state at a $50 \mathrm{~Hz}$ rate which is the validated rate for the model flown. 
Table 1 Initial Conditions for Simulated Routes

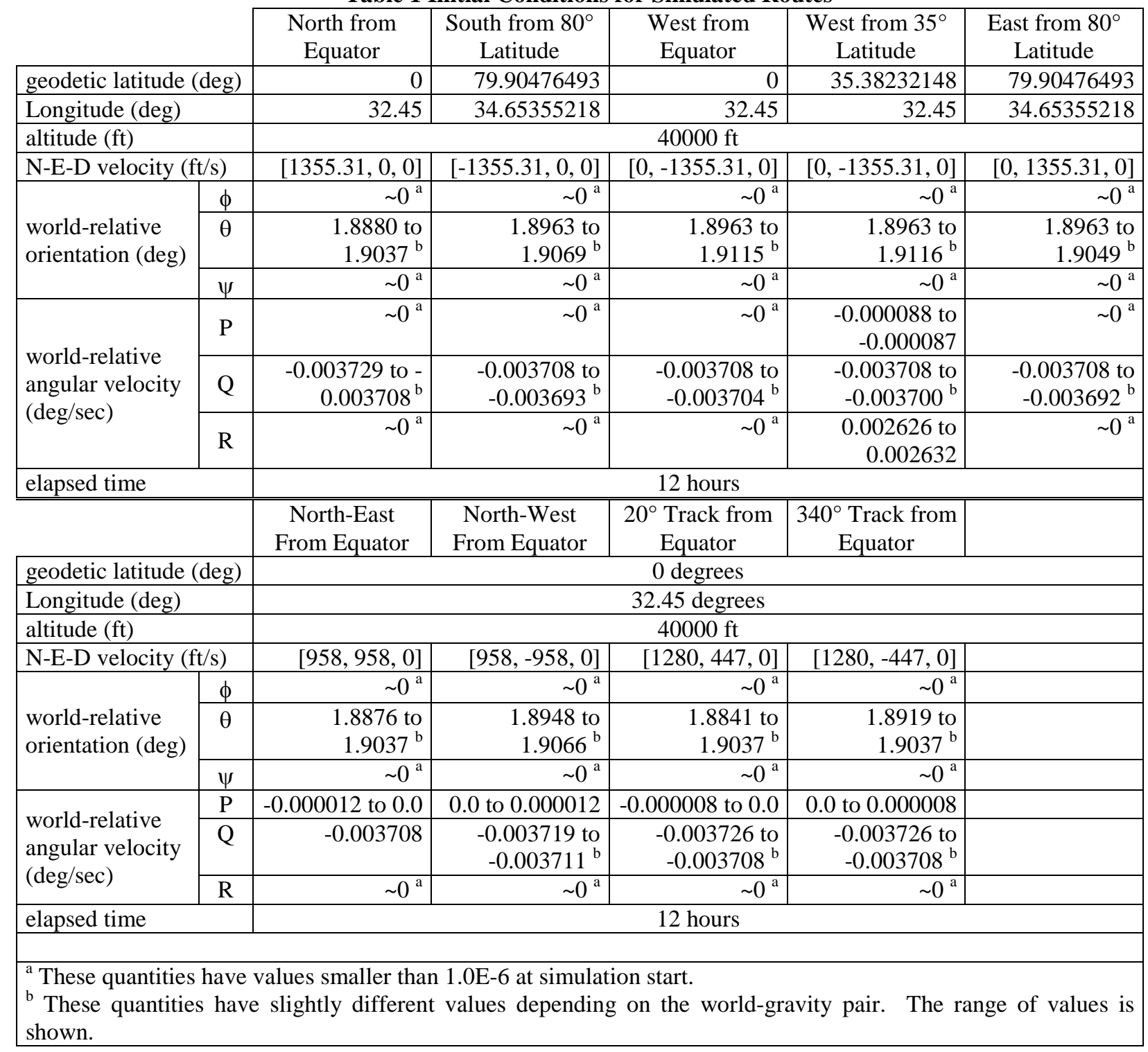

One difference between the subsonic and supersonic study is the treatment of lateral control surfaces. Both the subsonic and supersonic aircraft models produce residual roll and yaw moments at the scenario start and these moments differ between the world-gravity pairs due to differences in initial conditions (e.g. angle of attack, angular velocity). In the subsonic study, the lateral control surfaces (aileron and rudder) were left at the neutral position. Due to the subsonic aircraft's large moments of inertia, the subsonic study assumed that differences in the residual roll and yaw moments would bring negligible differences to the trajectories of the world-gravity pairs. As a result of keeping the lateral control surfaces in the neutral position, the subsonic aircraft flies a great circle route for the West from $35^{\circ}$ Latitude and East from $80^{\circ}$ Latitude scenarios rather than maintain latitude. Initial results in the supersonic study showed unexpected growth in the mid-route errors of some world-gravity pairs when compared to the subsonic results. When the lateral control surfaces were manipulated to cancel the initial roll and yaw accelerations, the mid-route errors decreased, demonstrating that residual roll and yaw moments can contribute significantly to relative error between trajectories. Note that the surfaces in this study were not manipulated to cancel the residual roll and yaw moments but to cancel the roll and yaw acceleration (which includes the cross product of the angular velocity and angular momentum). This is a restriction of LaSRS++ simulation which does not offer the option of canceling the moments directly. However, canceling the roll and yaw acceleration has nearly the same effect; the residual moments are reduced by three orders of magnitude or more. Manipulating the lateral control surfaces also generates added drag and side force. However, the drag is canceled as part of trimming the vehicle to zero acceleration 
in the x-body axis. Moreover, the aerodynamic side forces generated by the supersonic model represent lateral accelerations orders of magnitude lower than the Coriolis acceleration or the centrifugal acceleration (and the differences in lateral acceleration between world-gravity pairs are even smaller). As a result of constraining the initial angular acceleration to zero, the supersonic aircraft maintains latitude rather than fly great circle routes in the West from $35^{\circ}$ Latitude and East from $80^{\circ}$ Latitude scenarios.

Each of the nine routes was selected based on conditions with the potential to elicit different trajectory behaviors due to surface or gravity modeling. The North from equator route starts on the equator and flies due north. Since gravity changes with latitude, this route attempts to cover the gravity's full range of values over the Earth surface. The South from $80^{\circ}$ Latitude route starts at the latitude, at which the subsonic aircraft in Reference 4 ended its north flight from the equator, and flies due South from there. The south route examines whether the performance trends presented in North from Equator route hold when the route is reversed. The West from Equator route starts at the equator and flies due west in opposition to the Earth's rotation. Because the equatorial radius differs between the ellipsoid and sphere world models (and that difference will influence the results for the equatorial route), the west route was repeated at $35.38232148^{\circ}$ latitude where the ellipsoid and sphere surface have the same geocentric radius. This is the West from $35^{\circ}$ Latitude route. The East from $80^{\circ}$ Latitude route causes the aircraft to spend time north of $35^{\circ}$ latitude where the surface lies at a greater geocentric radius on the sphere than on the ellipsoid. The remaining routes look at tracks where the initial velocity has both a North and East component. The North-East from Equator scenarios looks at a route with a $45^{\circ}$ track and the North-West from Equator looks at the mirror route ( $-45^{\circ}$ track). These latter two scenarios produce trajectories that spend a majority of time below $35^{\circ}$ latitude. To produce trajectories with more equitable time above and below $35^{\circ}$ latitude, the $20^{\circ}$ Track from Equator and $340^{\circ}$ Track from Equator routes were created.

\section{A. Prior Work}

References 2, 4, and 5 lay the ground work for this study. In reference 2, the fidelity of the world-gravity pairs in reproducing gravity at the surface was analyzed. The analysis found that the rotating world-gravity pairs (except E/SE) produce a residual component of gravity that is tangent to the surface. For the E/PM and E/C world-gravity pairs, the surface mean of this residual tangent gravity was $0.01073 \mathrm{~m} / \mathrm{s}^{2}$ toward the pole. The S/PM and S/C worldgravity pairs produce slightly larger mean tangent gravity of $-0.01129 \mathrm{~m} / \mathrm{s}^{2}$, directed in the opposite direction toward the equator. The S/SE word-gravity pair produced the largest mean tangent gravity of $-0.02194 \mathrm{~m} / \mathrm{s}^{2}$, also directed toward the equator. However, reference 2 used the equivalence of geocentric and geodetic coordinates on the sphere world to directly input the geodetic coordinates into the symmetric ellipsoid model. Reference 5 demonstrated that, if the simulation forced the symmetric ellipsoid model to produce the same gravitation vector for the same geodetic coordinates (i.e. as if the geodetic coordinates relative to the sphere were at the same geocentric location as the geodetic coordinates relative to the ellipsoid), then the residual tangent gravity becomes negligible for the S/SE pair. Moreover, the position errors using this latter approach were an order of magnitude lower in most cases. As explained in section II.B.4, this is the approach used to model the S/SE world-gravity pair in this study.

References 4 and 5 established that the position errors of the reduced-fidelity, world-gravity pairs can be divided into contributions from residual tangent gravity, missing Coriolis acceleration, and reduced surface fidelity. ${ }^{4,5}$ This identification is possible because select world-gravity pairs differ from the reference E/SE pair only by these contributors. The E/PM and E/C world-gravity pairs isolate the effects of residual tangent gravity. The ENR/FA and ENR/C world-gravity pairs isolate the effects of missing Coriolis acceleration. The S/SE world gravity pair isolates the effect of reduced surface fidelity. Based on this prior work, the analysis in this paper also explains the position errors of the trajectories for each world-gravity pair using these three contributors.

The subsonic aircraft study in References 4 and 5 showed that the order of the world-gravity pairs by position error can depend on the route. However, some general trends did emerge for subsonic aircraft simulation. In all but one route, the S/SE gravity pair produced the lowest position error. Thus, when reducing fidelity by using a sphere model of the Earth, S/SE should be the first choice. When reducing the fidelity of the gravity or gravitation model, the non-rotating ENR/FA unexpectedly emerged as the best general choice. It outperformed or equaled the E/PM, $\mathrm{S} / \mathrm{PM}$, and SNR/FA pairs in all but one route (two routes if judging based on distance error at completion). SNR/FA was a good second choice; it outperformed or equaled the E/PM and S/PM world-gravity pairs in all but one route. The pairs using constant gravity or constant gravitation models did perform equally to counterparts using point-mass gravitation or free-air reduction gravity. However, this equality of performance was assumed to be an artifact of straight and level flight where the aircraft is initialized to cancel the initial gravity acceleration, so world-gravity pairs using point-mass gravitation and free-air reduction were given preference. One limitation of the subsonic air- 
craft study is that the missing Coriolis acceleration in the non-rotating world-gravity pairs is small at subsonic speeds. This paper, therefore, expands on this prior work by examining the same scenarios under supersonic speeds that generate larger Coriolis accelerations.

\section{Results}

Figure 1 through Figure 36 present the results of the simulations. The figures depict the geodetic latitude and longitude of the resulting vehicle trajectories. A plot from start-to-finish and a plot of travel in the last hour are both provided. (Note that the latitude or longitude trajectories of related world-gravity pairs may be nearly identical on a given route; therefore, some plot lines may be hidden behind others.) Altitude differences also appear among the trajectories (errors of up to 150 feet in some cases). However, those differences are tiny in comparison to the total position error and are not necessary to explain the trajectory differences among world-gravity pairs. Therefore, altitude data is not presented or discussed. The simulation results are further reduced to a series of position errors. To compute the position error, the geodetic coordinates of each run are transformed into a geocentric position vector using equations 4-14 and 4-15 in Reference 2. These equations relocate the geodetic coordinates onto the ellipsoid Earth so that geodetic coordinates of all trajectories are referenced to the same shape. The position vector associated with each world-gravity pair is subtracted by the position vector for the trajectory of the reference worldgravity pair, E/SE. The result is the position error relative to the E/SE trajectory. For each world-gravity pair, Table 2 through Table 10 present the maximum magnitude of the position error and the magnitude of the position error at the mid-point and end of the trajectory. The mid-point covers about the same distance as the subsonic aircraft study in References 4 and 5 allowing closer comparison with the subsonic results. Unfortunately, the fighter model used in this study did not have sufficient roll stability to run the subsonic scenarios of References 4 and 5; so direct comparison was not possible.

Table 2 North from Equator

\begin{tabular}{|l|r|r|r|}
\hline $\begin{array}{c}\text { World- } \\
\text { gravity } \\
\text { pair }\end{array}$ & $\begin{array}{c}\text { Max } \\
\text { distance } \\
\text { error (nmi) }\end{array}$ & $\begin{array}{c}\text { Mid-route } \\
\text { error (nmi) }\end{array}$ & $\begin{array}{c}\text { End-route } \\
\text { error (nmi) }\end{array}$ \\
\hline E/PM & 14.5600 & 13.7986 & 6.5176 \\
\hline E/C & 13.3829 & 13.0422 & 4.4244 \\
\hline S/SE & 10.2069 & 5.6658 & 1.3411 \\
\hline S/PM & 18.2387 & 16.9783 & 1.7369 \\
\hline S/C & 18.2286 & 16.9639 & 1.4740 \\
\hline ENR/FA & 131.4960 & 91.6451 & 86.9436 \\
\hline ENR/C & 131.4960 & 91.6451 & 86.9436 \\
\hline SNR/FA & 131.4748 & 91.8408 & 86.9381 \\
\hline SNR/C & 131.4748 & 91.8408 & 86.9381 \\
\hline
\end{tabular}

Table 4 West from Equator

\begin{tabular}{|l|r|r|r|}
\hline $\begin{array}{l}\text { World- } \\
\text { gravity } \\
\text { pair }\end{array}$ & $\begin{array}{c}\text { Max } \\
\text { distance } \\
\text { error (nmi) }\end{array}$ & $\begin{array}{c}\text { Mid-route } \\
\text { error (nmi) }\end{array}$ & $\begin{array}{c}\text { End-route } \\
\text { error (nmi) }\end{array}$ \\
\hline E/PM & 0.0000 & 0.0000 & 0.0000 \\
\hline E/C & 0.0000 & 0.0000 & 0.0000 \\
\hline S/SE & 10.7630 & 5.3815 & 10.7630 \\
\hline S/PM & 10.7630 & 5.3815 & 10.7630 \\
\hline S/C & 10.7630 & 5.3815 & 10.7630 \\
\hline ENR/FA & 0.0000 & 0.0000 & 0.0000 \\
\hline ENR/C & 0.0000 & 0.0000 & 0.0000 \\
\hline SNR/FA & 10.7630 & 5.3815 & 10.7630 \\
\hline SNR/C & 10.7630 & 5.3815 & 10.7630 \\
\hline
\end{tabular}

Table 3 South from $80^{\circ}$ Latitude

\begin{tabular}{|l|r|r|r|}
\hline $\begin{array}{c}\text { World- } \\
\text { gravity } \\
\text { pair }\end{array}$ & $\begin{array}{c}\text { Max } \\
\text { distance } \\
\text { error (nmi) }\end{array}$ & $\begin{array}{c}\text { Mid-route } \\
\text { error (nmi) }\end{array}$ & $\begin{array}{c}\text { End-route } \\
\text { error (nmi) }\end{array}$ \\
\hline E/PM & 17.3151 & 5.4713 & 17.3151 \\
\hline E/C & 15.0512 & 6.2722 & 15.0512 \\
\hline S/SE & 15.8964 & 5.7535 & 11.6581 \\
\hline S/PM & 22.6972 & 1.5836 & 22.1351 \\
\hline S/C & 22.6684 & 1.6314 & 22.1118 \\
\hline ENR/FA & 158.2397 & 69.4131 & 158.2397 \\
\hline ENR/C & 158.2397 & 69.4131 & 158.2397 \\
\hline SNR/FA & 158.4681 & 69.7151 & 158.4681 \\
\hline SNR/C & 158.4681 & 69.7151 & 158.4681 \\
\hline
\end{tabular}

Table 5 West from $35^{\circ}$ Latitude

\begin{tabular}{|l|r|r|r|}
\hline $\begin{array}{l}\text { World- } \\
\text { gravity } \\
\text { pair }\end{array}$ & $\begin{array}{c}\text { Max } \\
\text { distance } \\
\text { error (nmi) }\end{array}$ & $\begin{array}{c}\text { Mid-route } \\
\text { error (nmi) }\end{array}$ & $\begin{array}{c}\text { End-route } \\
\text { error (nmi) }\end{array}$ \\
\hline E/PM & 36.0163 & 33.0650 & 35.1581 \\
\hline E/C & 36.1331 & 33.1379 & 35.2861 \\
\hline S/SE & 22.2876 & 11.0036 & 22.2876 \\
\hline S/PM & 31.8080 & 31.5471 & 16.6030 \\
\hline S/C & 31.8070 & 31.5461 & 16.6019 \\
\hline ENR/FA & 85.7554 & 78.9152 & 83.8168 \\
\hline ENR/C & 85.6133 & 78.7874 & 83.6768 \\
\hline SNR/FA & 77.1841 & 74.5441 & 63.2158 \\
\hline SNR/C & 77.1841 & 74.5441 & 63.2158 \\
\hline
\end{tabular}


Table 6 East from $80^{\circ}$ Latitude

\begin{tabular}{|l|r|r|r|}
\hline $\begin{array}{l}\text { World- } \\
\text { gravity } \\
\text { pair }\end{array}$ & $\begin{array}{c}\text { Max } \\
\text { distance } \\
\text { error (nmi) }\end{array}$ & $\begin{array}{c}\text { Mid-route } \\
\text { error (nmi) }\end{array}$ & $\begin{array}{c}\text { End-route } \\
\text { error (nmi) }\end{array}$ \\
\hline E/PM & 9.6906 & 4.9791 & 9.6906 \\
\hline E/C & 9.5956 & 4.9728 & 9.5956 \\
\hline S/SE & 41.5037 & 20.8454 & 41.5037 \\
\hline S/PM & 38.1037 & 19.2068 & 29.9096 \\
\hline S/C & 38.2151 & 19.3844 & 30.4821 \\
\hline ENR/FA & 50.7124 & 32.7220 & 50.7124 \\
\hline ENR/C & 51.1073 & 32.9567 & 51.1073 \\
\hline SNR/FA & 92.1578 & 50.5433 & 92.1578 \\
\hline SNR/C & 92.1578 & 50.5433 & 92.1578 \\
\hline
\end{tabular}

Table 7 North East from Equator

\begin{tabular}{|l|r|r|r|}
\hline $\begin{array}{l}\text { World- } \\
\text { gravity } \\
\text { pair }\end{array}$ & $\begin{array}{c}\text { Max } \\
\text { distance } \\
\text { error (nmi) }\end{array}$ & $\begin{array}{c}\text { Mid-route } \\
\text { error (nmi) }\end{array}$ & $\begin{array}{c}\text { End-route } \\
\text { error (nmi) }\end{array}$ \\
\hline E/PM & 34.2734 & 24.4469 & 23.2783 \\
\hline E/C & 34.6887 & 24.7003 & 23.7825 \\
\hline S/SE & 84.6448 & 15.7385 & 84.6448 \\
\hline S/PM & 109.0427 & 41.5970 & 109.0427 \\
\hline S/C & 108.7679 & 41.3429 & 108.7679 \\
\hline ENR/FA & 90.1542 & 63.6732 & 58.4977 \\
\hline ENR/C & 90.2006 & 63.6838 & 58.6030 \\
\hline SNR/FA & 57.4065 & 47.3042 & 28.1890 \\
\hline SNR/C & 57.4065 & 47.3042 & 28.1890 \\
\hline
\end{tabular}

Table $920^{\circ}$ Track from Equator

\begin{tabular}{|l|r|r|r|}
\hline $\begin{array}{l}\text { World- } \\
\text { gravity } \\
\text { pair }\end{array}$ & $\begin{array}{c}\text { Max } \\
\text { distance } \\
\text { error (nmi) }\end{array}$ & $\begin{array}{c}\text { Mid-route } \\
\text { error (nmi) }\end{array}$ & $\begin{array}{c}\text { Final } \\
\text { distance } \\
\text { error (nmi) }\end{array}$ \\
\hline E/PM & 25.3766 & 19.6902 & 17.1502 \\
\hline E/C & 24.9597 & 19.4712 & 17.0537 \\
\hline S/SE & 52.0461 & 10.0920 & 52.0461 \\
\hline S/PM & 69.9709 & 29.9130 & 69.9709 \\
\hline S/C & 69.6367 & 29.6481 & 69.6367 \\
\hline ENR/FA & 123.0233 & 86.1103 & 80.5874 \\
\hline ENR/C & 123.0526 & 86.1170 & 80.6541 \\
\hline SNR/FA & 99.9588 & 76.0094 & 26.4335 \\
\hline SNR/C & 99.9588 & 76.0094 & 26.4335 \\
\hline
\end{tabular}

Table 8 North West from Equator

\begin{tabular}{|l|r|r|r|}
\hline $\begin{array}{l}\text { World- } \\
\text { gravity } \\
\text { pair }\end{array}$ & $\begin{array}{c}\text { Max } \\
\text { distance } \\
\text { error (nmi) }\end{array}$ & $\begin{array}{c}\text { Mid-route } \\
\text { error (nmi) }\end{array}$ & $\begin{array}{c}\text { Final } \\
\text { distance } \\
\text { error (nmi) }\end{array}$ \\
\hline E/PM & 35.0934 & 24.7807 & 24.8254 \\
\hline E/C & 35.0740 & 24.6755 & 24.9937 \\
\hline S/SE & 83.0562 & 16.0834 & 83.0562 \\
\hline S/PM & 109.1310 & 42.1775 & 109.1310 \\
\hline S/C & 109.2654 & 42.2887 & 109.2654 \\
\hline ENR/FA & 93.8799 & 64.7090 & 65.4101 \\
\hline ENR/C & 93.8341 & 64.6991 & 65.3124 \\
\hline SNR/FA & 147.8046 & 80.6996 & 147.2417 \\
\hline SNR/C & 147.8046 & 80.6996 & 147.2417 \\
\hline
\end{tabular}

Table $10340^{\circ}$ Track from Equator

\begin{tabular}{|l|r|r|r|}
\hline $\begin{array}{l}\text { World- } \\
\text { gravity } \\
\text { pair }\end{array}$ & $\begin{array}{c}\text { Max } \\
\text { distance } \\
\text { error (nmi) }\end{array}$ & $\begin{array}{c}\text { Mid-route } \\
\text { error (nmi) }\end{array}$ & $\begin{array}{c}\text { Final } \\
\text { distance } \\
\text { error (nmi) }\end{array}$ \\
\hline E/PM & 22.5164 & 18.6398 & 13.7988 \\
\hline E/C & 21.8153 & 18.1716 & 13.3465 \\
\hline S/SE & 53.5580 & 11.6329 & 53.5580 \\
\hline S/PM & 67.6909 & 29.7612 & 67.6909 \\
\hline S/C & 67.9353 & 29.9365 & 67.9353 \\
\hline ENR/FA & 125.2361 & 86.7257 & 85.3056 \\
\hline ENR/C & 125.2088 & 86.7195 & 85.2438 \\
\hline SNR/FA & 153.3346 & 96.8534 & 136.7235 \\
\hline SNR/C & 153.3346 & 96.8534 & 136.7235 \\
\hline
\end{tabular}

The position errors in the trajectories are explained by analyzing the three major contributors of position error: residual tangent gravity (present in rotating world gravity pairs), Coriolis acceleration (not present in non-rotating world-gravity pairs), or reduced surface fidelity (present in sphere world-gravity pairs). ${ }^{4}$ Five of the world-gravity pairs isolate the impact of each contributor. The E/PM and E/C world-gravity pairs isolate the effects of residual tangent gravity. The ENR/FA and ENR/C world-gravity pairs isolate the effects of missing Coriolis acceleration. The S/SE pair isolates the effects of reduced surface fidelity. The remaining subsections examine the impact of those contributors on each route. The discussion focuses on the mid-route and final position errors of the trajectories but the maximum position error is acknowledged when it shows a different trend.

\section{A. North from Equator}

The North from Equator route begins at the equator and flies due North. For this route, Figure 1 through Figure 4 show the latitude and longitude travel of the aircraft, and Table 2 presents the trajectory errors for each reducedfidelity, world-gravity pair. The missing Coriolis acceleration, embodied in the ENR/FA and ENR/C trajectories, 
contributes the largest error on this route. The vehicle's track produces Coriolis acceleration that is increasingly to the east and south with latitude in the first half of the route and is decreasingly south and west in the second half of the route. Figure 2 shows that the non-rotating world-gravity trajectories maintain initial longitude (or its converse on the other side of the Earth); however, the rotating world-gravity trajectories curve east from the initial longitude as they approach the pole due to Coriolis acceleration. As the aircraft turns south, the Coriolis acceleration flips west, and the rotating world-gravity pairs converges back toward the converse longitude. As a result, the ENR/FA and ENR/C trajectories lie $0.109^{\circ}$ north and $8.836^{\circ}$ west of the E/SE trajectory at the mid-point, but reduce to $0.069^{\circ}$ north and $1.532^{\circ}$ at completion. The reduced surface fidelity embodied in the S/SE pair generates a smaller trajectory error than the residual tangent gravity of the $\mathrm{E} / \mathrm{PM}$ and $\mathrm{E} / \mathrm{C}$ pairs. The S/SE trajectory is $0.089^{\circ}$ south and $0.179^{\circ}$ west at the midpoint and $0.001^{\circ}$ south and $0.024^{\circ}$ east at completion. The E/PM pair, whose tangent gravity is to the north, is $0.228^{\circ}$ north and 0.105 east at the midpoint and is $0.097^{\circ}$ south and $0.053^{\circ}$ east at completion. Though both are affected only by tangent gravity, the E/PM and E/C trajectories are separated $0.75 \mathrm{nmi}$ to $2.1 \mathrm{nmi}$ in Table 2; this route and the South from $80^{\circ}$ Latitude are the only routes were a significant difference is seen between the lower fidelity gravitation models on the same world model. The reason is that E/PM is the only pair with a reduced fidelity gravity/gravitation model where the vertical gravity at 40,000 feet will change with latitude. Because the contribution to Coriolis acceleration is an order of magnitude larger than the contribution due to reduced surface fidelity, the errors of the SNR/FA and SNR/C trajectories are largely unchanged compared to ENR/FA and ENR/C. In the $\mathrm{S} / \mathrm{PM}$ and S/C pairs whose tangent gravity is directed south, residual tangent gravity and reduced surface fidelity combine to generate larger south and west drifts at mid-point and generate smaller south and west drifts at completion. The S/PM trajectory is $0.278^{\circ}$ south and $0.218^{\circ}$ west of the E/SE trajectory at midpoint and is $0.012^{\circ}$ south and $0.028^{\circ}$ west of the E/SE trajectory at completion. The S/PM and S/C trajectories have larger maximum and midroute position errors than the E/PM and E/C trajectories but lower position errors at completion.

\section{B. South from $80^{\circ}$ Latitude}

The South from $80^{\circ}$ Latitude route is the north route of Reference 4 in reverse. For this route, Figure 5 through Figure 8 show the latitude and longitude travel of the aircraft, and Table 3 presents the trajectory errors for each reduced-fidelity, world-gravity pair. Similar to the North from Equator route, the missing Coriolis acceleration contributes the largest trajectory error. On this route, the aircraft's track produces a westward but decreasing Coriolis acceleration in the northern hemisphere and an eastward and increasing Coriolis acceleration in the southern hemisphere. Towards the end of the route, a northward Coriolis acceleration is generated as the aircraft turns east. Again, the non-rotating word-gravity trajectories hold longitude while the rotating world-gravity trajectories drift west then east of the initial longitude. By mid-route, the ENR/FA and ENR/C trajectories are $1.153^{\circ}$ east and less than $0.11^{\circ}$ north of the E/SE trajectory. By completion, the trajectory error grows to $15.37^{\circ}$ west and less than $0.39^{\circ}$ south. The contributions from tangent gravity and reduced surface fidelity show mixed results along the route. As with the North from Equator route, the E/PM and E/C trajectories errors differ by $-0.8 \mathrm{nmi}$ at mid-route and $+2.25 \mathrm{nmi}$ at completion showing a difference in the contributors affected only by tangent gravity. Again, the value of gravity at the 40,000 ft altitude changes with latitude under E/PM while the gravity remains constant under the E/C model. At the mid-point where the aircraft crosses the equator, the E/PM trajectory is at $0.091^{\circ}$ north and $<0.001^{\circ}$ east of E/SE. At completion, the E/PM trajectory lies $0.281^{\circ}$ south and $0.345^{\circ}$ east of the E/SE trajectory. The error contribution from reduced surface fidelity is comparable to the contribution from tangent gravity for the mid-route and maximum errors but is lower for the error at completion. The S/SE trajectory is $0.096^{\circ}$ north and $<0.001^{\circ}$ east of $\mathrm{E} / \mathrm{SE}$ at mid-route; at completion, the trajectory lies $0.160^{\circ}$ north and $0.622^{\circ}$ west at completion. As with the north route, the error contributions from missing Coriolis acceleration are so much larger than those from reduced surface fidelity that the errors of the SNR/FA and SNR/C trajectories do not differ appreciably from the SNR/FA and SNR/C trajectories. In the S/PM and S/C trajectories, residual tangent gravity (which is opposite that of E/PM) and reduced surface fidelity combine to nearly cancel north drift at mid-point and generate larger north and west drifts at completion. The result is that the S/PM and S/C trajectories lie up to $0.027^{\circ}$ south and $0.005^{\circ}$ east of $\mathrm{E} / \mathrm{SE}$ at the midpoint and lie $0.345^{\circ}$ north and up to $0.735^{\circ}$ west at completion. The S/PM and S/C trajectories have smaller position errors than $\mathrm{E} / \mathrm{PM}$ and $\mathrm{E} / \mathrm{C}$ at the mid-point but larger errors at completion.

\section{West from Equator}

This route starts on the equator and flies due west. For this route, Figure 9 through Figure 12 show the latitude and longitude travel of the aircraft, and Table 4 presents the trajectory errors for each reduced-fidelity, world-gravity pair. At the equator, none of the world-gravity pairs produce a residual tangent gravity. Moreover, when traveling due east or west on the equator, the Coriolis acceleration is directed only along the Down axis of the North-EastDown coordinate system. Since the aircraft is trimmed to a vertical acceleration of zero, differences in Coriolis ac- 
celeration, like differences in gravity, are offset by differences in initial pitch angle that give rise to a counteracting aerodynamic lift. Therefore, the only contributor left to influence aircraft trajectories is reduced surface fidelity. As seen in Table 4, the position errors for the ellipsoid world-gravity pairs are nearly zero and the sphere world-gravity pairs have the same error of $10.7630 \mathrm{nmi}$. The error is due to differences in the equatorial radii of the sphere and ellipsoid. The ellipsoid model uses the mean equatorial radius. The sphere model uses the radius of a sphere with equal surface area to the ellipsoid; this radius is smaller than the mean equatorial radius (but larger than the polar radius). Using simple geometry, the nearly $9600 \mathrm{nmi}$ travel of the aircraft will traverse $\sim 160.01^{\circ}$ of longitude on the ellipsoid (radius $=6378137 \mathrm{~m}$ ) and $\sim 160.18^{\circ}$ of longitude on the sphere (radius $=6371007 \mathrm{~m}$ ). The difference of $0.179^{\circ}$ of longitude equals a distance of $10.7428 \mathrm{nmi}$ along the equator of the ellipsoid.

\section{West from $35^{\circ}$ Latitude}

This route begins at the latitude where the geocentric radius of the ellipsoid surface equals that of the sphere surface. The initial velocity of the aircraft is due west. For this route, Figure 13 through Figure 16 show the latitude and longitude travel of the aircraft, and Table 5 presents the trajectory errors for each reduced-fidelity, world-gravity pair. Missing Coriolis acceleration contributes the largest error for this route. With the aircraft traveling west, the Coriolis acceleration is directed north. Figure 13 does show that the non-rotating world-gravity pairs produce trajectories that lie south of those produced by rotating world-gravity pairs. But, all world gravity pairs curve back toward the initial latitude at completion. At the mid-point, the ENR/FA and ENR/C trajectories lie up to $1.120^{\circ}$ south and $0.841^{\circ}$ east of the E/SE pair. By completion, the ENR/FA trajectory is up to $0.016^{\circ}$ south and $1.705^{\circ}$ east. The tangent gravity generates a larger error than the reduced surface fidelity. The E/PM and E/C trajectories, which represent the contribution of tangent gravity, have a latitude error of up to $0.468^{\circ}$ north and a longitude error of up to $0.361^{\circ}$ west at the mid-point; at completion, the trajectory lies up to $0.003^{\circ}$ south and up to $0.718^{\circ}$ west of E/SE. The S/SE trajectory, which represents the error contribution from reduced surface fidelity, lies $0.007^{\circ}$ north and $0.225^{\circ}$ west of E/SE at the mid-point; at completion, it is $0.003^{\circ}$ south and $0.453^{\circ}$ west of E/SE. The S/SE error is largely due to a west displacement. This displacement is caused by the fact that, even though the geocentric radii of the surface are equal length at this latitude, the radii of the latitude circle are not. The cross-sectional radius of the ellipsoid at this latitude (5205982 $\mathrm{m}$ ) is larger than the cross sectional radius on the sphere (5194324 $\mathrm{m})$. This difference alone would contribute to a west displacement of $\sim 21.586 \mathrm{nmi}$ at completion, comparable to the total distance error of $22.2876 \mathrm{nmi}$ for S/SE. For the SNR/FA and SNR/C trajectories, the missing Coriolis acceleration and reduced surface fidelity combine to decrease the east drift throughout the route; only missing Coriolis acceleration contributes to the latitude error. At the mid-point, the SNR/FA and SNR/C trajectories lie up to $1.130^{\circ}$ south and $0.629^{\circ}$ east of E/SE; at completion, the trajectories are up to $0.013^{\circ}$ south and $1.286^{\circ}$ east of E/SE. The result is that the SNR/FA and SNR/C trajectories have lower position errors than ENR/FA and ENR/C trajectories. For the S/PM and S/C pairs, the west error contribution from reduced surface fidelity reduces the east error contribution from tangent gravity; only tangent gravity contributes to the latitude error. The S/PM and S/C trajectories lie $0.509^{\circ}$ south and $0.162^{\circ}$ east of $\mathrm{E} / \mathrm{SE}$ at the midpoint; at completion, the trajectories lie $0.006^{\circ}$ south and $0.338^{\circ}$ east of $\mathrm{E} / \mathrm{SE}$. As a result, the S/PM and S/C trajectories have lower position errors than the E/PM and E/C trajectories.

\section{E. East from $80^{\circ}$ Latitude}

This route examines trajectories that start at an altitude where the geocentric radius to the surface is smaller on the ellipsoid than the sphere model. The vehicle initial velocity is due east. For this route, Figure 17 through Figure 20 show the latitude and longitude travel of the aircraft, and Table 6 presents the trajectory errors for each reducedfidelity, world-gravity pair. The missing Coriolis acceleration remains the largest contributor to trajectory error. The initial Coriolis acceleration is directed south in this scenario and Figure 17 shows that the trajectories of the non-rotating world-gravity pairs initially lie north of the E/SE trajectory. However, all trajectories oscillate about the initial latitude throughout the run. The ENR/FA trajectory lies $0.351^{\circ}$ north and $2.128^{\circ}$ east of E/SE at the midroute; at completion, the ENR/FA trajectory lies $0.084^{\circ}$ north and $4.702^{\circ}$ east of E/SE. The reduced surface fidelity generates a larger error contribution than the tangent gravity. On this route the aircraft maintains latitude as shown in Figure 17; therefore, the error contribution of reduced surface fidelity is due to the radial differences of the circular cross section at the $80^{\circ}$ latitude line. Though the sphere has a larger geocentric radius to the surface, the cross section radius is larger on the ellipsoid $(1121636 \mathrm{~m})$ than the sphere $(1116741 \mathrm{~m})$. The larger geocentric radius of the sphere is largely manifest as a larger Earth z-axis height for the cross section. The smaller sphere radius generates an eastward displacement of S/SE, as shown in Figure 20. Using simple geometry, the expected east displacement at the end of the run is $\sim 2.154 \mathrm{nmi}$, comparable to the $41.5037 \mathrm{nmi}$ error of the S/SE trajectory. In terms of geodetic coordinates, the S/SE trajectory lies $0.008^{\circ}$ south and $1.752^{\circ}$ east of E/SE at the mid-point and lies $0.082^{\circ}$ north and $3.840^{\circ}$ east at completion. The $\mathrm{E} / \mathrm{PM}$ and $\mathrm{E} / \mathrm{C}$ trajectories indicate that the tangent gravity generates errors of 
$0.075^{\circ}$ north and up to $0.175^{\circ}$ east at the midpoint; at completion, the trajectories lie up to $0.032^{\circ}$ north and 0.882 degrees east of E/SE. For the SNR/FA and SNR/C trajectories, the contributions of missing Coriolis acceleration and reduced surface fidelity are additive. The SNR/FA and SNR/C trajectories lie up to $0.347^{\circ}$ north and $3.950^{\circ}$ east of $\mathrm{E} / \mathrm{SE}$ at the mid-point; at completion, the trajectories lie $0.144^{\circ}$ north and up to $8.618^{\circ}$ east of $\mathrm{E} / \mathrm{SE}$. As a result, the SNR/FA and SNR/C trajectories have the largest position errors. The S/PM and S/C trajectories show that the contributions of tangent gravity and reduced surface fidelity reduce the east errors from reduced surface fidelity; for latitude errors, the combination should generate an increased south error at the midpoint and a decreased north error at completion. The S/PM and S/C trajectories lie up to $0.092^{\circ}$ south and up to $1.561^{\circ}$ east of E/SE at the midpoint; at completion, the trajectories lie up to $0.048^{\circ}$ north and $2.770^{\circ}$ east of E/SE. S/PM and S/C produce lower position errors than the S/SE trajectory but have larger errors than the E/PM and E/C trajectories.

\section{F. North-East from Equator and North-West from Equator}

The North-East from Equator and North-West from Equator routes are discussed together because they have mirrored initial conditions. The North-East from Equator route takes a $45^{\circ}$ track and the North-West from Equator route takes a $-45^{\circ}$ track. For both routes, the East component of Coriolis acceleration points east in the first half of the trajectory. As the aircraft follows a great circle route, the velocity turns more easterly (or westerly) toward the middle of the trajectory, and the Coriolis acceleration will turn to more southerly (or northerly). In the last half of the trajectory, the aircraft turns to the south and the East component of Coriolis acceleration flips to the west.

Figure 21 and Figure 22 show the latitude and longitude trends in the trajectories of the North-East from Equator route. In this route, the S/SE trajectory visibly departs from the E/SE trajectory. Reduced surface fidelity causes the S/SE trajectory to drift south $0.258^{\circ}$ and drift east $0.058^{\circ}$ at the midpoint; at completion, the trajectory lies $1.112^{\circ}$ south and $0.894^{\circ}$ west at completion. The S/SE error accelerates substantially in the last half of the route. This arises from the interplay of curvature differences in latitude and longitude between the sphere and ellipsoid when the aircraft bends back to the south. The cross-section radius for each line of latitude declines more slowly on the ellipsoid than on the sphere. When traveling east or west, the sphere worlds will accumulate distance error due to its smaller cross section radius, i.e. larger curvature. For each $1000 \mathrm{nmi}$ traveled east or west, that error is $1.117 \mathrm{nmi}$ at the equator and grows to $4.477 \mathrm{nmi}$ near the pole. In this route, the aircraft travels continuously east so it continually accumulates error to the east. The sphere also accumulates error on its north-south travel because the curvature along lines of longitude differs between the sphere and ellipsoid. The curvature is greater (i.e. more latitude traversed per nautical mile) on the ellipsoid at latitudes less than $48^{\circ}$ and is smaller at latitudes greater than $48^{\circ}$. Thus, error accumulated below $48^{\circ}$ latitude is diminished with travel above $48^{\circ}$ latitude and vice-versa. In this route, the $\mathrm{E} / \mathrm{SE}$ and S/SE trajectories curve south at the same time. However, due to the differences in curvature, the S/SE trajectory curves at a latitude south of the E/SE trajectory and, because the curvature along lines of latitude increase with latitude, the E/SE pair accelerates past S/SE as the track turns east (or west) at the bend of the trajectory. The rapid separation in latitude and longitude at the bend is amplified as the aircraft travels south. The residual tangent gravity causes the $\mathrm{E} / \mathrm{PM}$ and $\mathrm{E} / \mathrm{C}$ trajectories to drift north up to $0.409^{\circ}$ and drift east up to $0.067^{\circ}$ at the midpoint; the trajectories drift up to $0.263^{\circ}$ north and $0.313^{\circ}$ east at completion. Residual tangent gravity causes drift in the opposite direction for the S/PM and S/C trajectories. Thus the combined effects of tangent gravity and reduced surface fidelity indicate a large south drift at both the midpoint and at completion, small east-west drift at midpoint, and a large west drift at completion. In fact, the S/PM and S/C trajectories drift south by up to $0.692^{\circ}$ at midpoint and $1.418^{\circ}$ at completion. These trajectories also drift east by up to $0.027^{\circ}$ at mid-point and drift west by up to $1.169^{\circ}$ at completion. As predicted by the direction of the Coriolis acceleration, the ENR/FA and ENR/C trajectories drift north and west of the E/SE trajectory by up to $1.044^{\circ}$ and $0.252^{\circ}$ respectively at mid-point and drift north and east by up to $0.710^{\circ}$ and $0.692^{\circ}$ degrees respectively at completion. Because the SNR/FA and SNR/C trajectories experience drift due to a combination of reduced surface fidelity and missing Coriolis acceleration, these trajectories have a reduced north and west drift of up to $0.775^{\circ}$ and $0.190^{\circ}$ respectively at midpoint. At completion, these trajectories have a lower south drift of up to $0.402^{\circ}$ and a lower west drift of up to $0.251^{\circ}$ (compared to the negative of the ENR/FA errors). Table 7 shows the resulting position errors. The missing Coriolis acceleration (ENR/FA and ENR/C) contributes the largest error except at completion where reduced surface fidelity (S/SE) is the largest contributor. The tangent gravity is the smallest contributor except at the mid-route where the reduced surface fidelity (S/SE) is the smallest contributor. The interplay among the contributors to position error produces mixed trends for sphere world-gravity pairs. S/PM and S/C produce lower errors than SNR/FA and SNR/C only at the mid-route. The maximum and at-completion errors for S/PM and S/C are the highest among world-gravity pairs displacing the non-rotating world-gravity pairs as the worst performers. Furthermore, SNR/FA and SNR/C at completion are the second lowest due to the counteracting contributions of reduced surface fidelity and missing Coriolis acceleration. 
Compared to the North-East from equator route, Figure 27 and Figure 28 show some mirrored trends in the North-West from Equator route, but the interplay among error contributors produces different results for the SNR/FA and SNR/C pairs. Reduced surface fidelity causes the S/SE trajectory to drift south by $0.264^{\circ}$ but, mirroring the North-East from Equator route, drifts $0.062^{\circ}$ west at the midpoint. At completion, the trajectory drifts $1.050^{\circ}$ south and $0.934^{\circ}$ east. Again the errors from reduced surface fidelity accelerate in the second half of the route. The Coriolis acceleration is perpendicular to the velocity vector. Therefore, the aircraft velocity that is mirrored from east to west produces a Coriolis acceleration that is mirrored from south to north. The East component of Coriolis acceleration, however, remains east in the first half of the trajectory and west in the second half. As predicted by the direction of the Coriolis acceleration, the ENR/FA and ENR/C trajectories drift south of the E/SE trajectory by up to $1.061^{\circ}$ and drift west by up to $0.255^{\circ}$ at the midpoint; at completion, the drift is up to $0.745^{\circ}$ south and $0.823^{\circ}$ east. The tangent gravity causes the E/PM and E/C trajectories to drift north by up to $0.410^{\circ}$ and, in mirror to the NorthEast from Equator route, drift west by up to $0.066^{\circ}$ at the mid-point. At completion, the drift is up to $0.261^{\circ}$ north and up to $0.349^{\circ}$ west. Acting in the opposite direction, the tangent gravity induces a south drift and east drift in the $\mathrm{S} / \mathrm{PM}$ and S/C trajectories. Again, the tangent gravity and surface modeling error combine to produce an increased southern drift of up to $0.703^{\circ}$ at mid-point (up to $1.366^{\circ}$ at completion), a near-zero drift of up to $0.038^{\circ}$ west at mid-point, and an increased east drift of up to $1.245^{\circ}$ at completion. The drift in the SNR/FA and SNR/C trajectories is a combination of missing Coriolis acceleration and reduced surface fidelity. Unlike the North-East from Equator route, the south drift due to missing Coriolis acceleration in the North-West from Equator route adds to the south drift due to reduced surface fidelity. Moreover, the initial east-west drift due to reduced surface fidelity combines with the east-west drift due to missing Coriolis acceleration. The result is that the SNR/FA and SNR/C trajectories drift south by an increased $1.323^{\circ}$ at mid-point $\left(1.806^{\circ}\right.$ at completion), drift west by an increased $0.315^{\circ}$ at mid-point, and drift east by an increased $1.717^{\circ}$ at completion. Table 8 shows the resulting position errors. The trends are largely the same as for the North-East from Equator route with the exception of the SNR/FA and SNR/C trajectories. The SNR/FA and SNR/C produce the largest trajectory errors over the route due to the fact that contributions from the missing Coriolis acceleration and reduced surface fidelity combine rather than counteract.

\section{G. $20^{\circ}$ Track from Equator and $340^{\circ}$ Track from Equator}

The $20^{\circ}$ Track from Equator and $340^{\circ}$ Track from Equator routes are another pair of routes with mirrored initial conditions. These routes attempt to produce trajectories that spend equal time each region where the geocentric radius of the sphere surface is smaller and larger than the geocentric radius of the ellipsoid surface. This was approximately true in the subsonic study, but, in this supersonic study, the aircraft spends only about four hours in the region where the geocentric radius of the ellipsoid surface is larger and the remaining eight hours in the region where the geocentric radius of the ellipsoid surface is smaller. Like the previous two routes, the Coriolis acceleration will have both a North and East component in these routes. For approximately the first half of the route, the Coriolis acceleration is directed east and south for the $20^{\circ}$ Track from Equator route and directed east and north for the $340^{\circ}$ Track from Equator route. In the second half of the route, the Coriolis acceleration is directed west and south for the $20^{\circ}$ Track from Equator route and directed west and north for the $340^{\circ}$ Track from Equator route. The East component will predominate early in the route, but, as the trajectory turns east (or west) toward the middle of the route, the North component will become dominant.

Figure 29 through Figure 32 show the latitude and longitude of the trajectories for the $20^{\circ}$ Track from Equator route. The reduced surface fidelity causes the S/SE trajectory to drift $0.167^{\circ}$ south and $0.006^{\circ}$ west at mid route and drift $0.350^{\circ}$ south and $0.833^{\circ}$ west at completion. As with the North-East from Equator route and its mirror, the error of the S/SE trajectory accelerates in the second half of the trajectory due to the interplay of latitude and longitude errors as the aircraft bends around to the south. Residual tangent gravity causes the E/PM and E/C trajectories to drift north by up to $0.317^{\circ}$ and drift east by up to $0.205^{\circ}$ at mid-point; at completion, the trajectories drift up to $0.052^{\circ}$ north and up to $0.300^{\circ}$ east. Thus, the affect of residual tangent gravity on S/PM and S/C trajectories is to drive the trajectories to the south and west. The south and west drift for theses trajectories is driven further by the effect of reduced surface fidelity. The S/PM and S/C trajectories drift up to $0.494^{\circ}$ south and $0.100^{\circ}$ west at midpoint and drift up to $0.471^{\circ}$ south and $1.119^{\circ}$ west at completion. As predicted by Coriolis acceleration, the ENR/FA and ENR/C trajectories drift to the north by $1.286^{\circ}$ and west by $1.651^{\circ}$ at mid-point. By completion, the trajectories drift $0.492^{\circ}$ north and up to $1.316^{\circ}$ east. In the SNR/FA and SNR/C trajectories, the effects of Coriolis acceleration and reduced surface fidelity combine to decrease the drift north throughout the route and decrease the drift east at completion. At the mid-point, the north drift is $1.101^{\circ}$ and the west drift is $1.626^{\circ}$. At completion, the north drift is $0.158^{\circ}$ and the west drift is $0.432^{\circ}$. Table 9 shows the resulting position errors. The missing Coriolis acceleration (ENR/FA and ENR/C) contributes the largest error. The tangent gravity is the smallest contributor 
except at the mid-route where the reduced surface fidelity (S/SE) is the smallest contributor. The interplay among the contributors to position error produces mixed trends for sphere world-gravity pairs. S/PM and S/C consistently produce larger errors than other rotating world-gravity pairs but lower errors than the non-rotating world gravity pairs except at completion where SNR/FA and SNR/C have lower errors. The SNR/FA and SNR/C trajectories have the second-highest errors except at completion where the error drops to the second lowest due to the counteracting contributions of reduced surface fidelity and missing Coriolis acceleration.

Figure 33 through Figure 36 show the latitude and longitude of trajectories on the $340^{\circ}$ Track from Equator route. The S/SE trajectory again drifts up to $0.192^{\circ}$ south in mid-route and $0.301^{\circ}$ south at completion. The S/SE trajectory also drifts up to $0.054^{\circ}$ west at mid-point and $0.887^{\circ}$ east at completion, mirroring the east-west drift under the $20^{\circ}$ Track from equator route. Thus, reduced surface fidelity causes drift south and, in the second half of the route, east drift. The $\mathrm{E} / \mathrm{PM}$ and $\mathrm{E} / \mathrm{C}$ trajectories show that residual tangent gravity causes a drift north up to $0.300^{\circ}$ at midpoint and minor north-south drift at completion. (E/PM is $0.026^{\circ}$ south and $\mathrm{E} / \mathrm{C}$ is $0.012^{\circ}$ north.) The trajectories drift up to $0.211^{\circ}$ west at mid-point and $0.242^{\circ}$ west at completion (mirroring the east drift in $20^{\circ}$ Track from Equator route). The tangent gravity for S/PM and S/C is in the opposite direction and drives those trajectories south and east. In the S/PM and S/C trajectories, the combined effects of residual tangent gravity and reduced surface fidelity push the trajectory up to $0.496^{\circ}$ south and up to $0.030^{\circ}$ east at mid-point. At completion, the trajectories drift up to $0.385^{\circ}$ south and $1.123^{\circ}$ east. As predicted by the Coriolis acceleration, the ENR/FA and ENR/C trajectories drift up to $1.282^{\circ}$ degrees south and $1.835^{\circ}$ west at mid-point. The trajectories drift $0.403^{\circ}$ south and $1.438^{\circ}$ east at completion. In the SNR/FA and SNR/C trajectories, the effect of Coriolis acceleration and surface modeling error combine to increase the south drift throughout the route and increase the east drift at completion. The SNR/FA and SNR/C directories drift $1.463^{\circ}$ south and $1.855^{\circ}$ west at the mid-point. At completion, the trajectories drift $0.722^{\circ}$ south and $2.278^{\circ}$ east. Table 10 presents the resulting position errors. The trends are the same as the $20^{\circ}$ Track from Equator route with one exception. The SNR/FA and SNR/C routes produce the largest error throughout the route.

\section{Conclusions}

This study investigates the impact of world-gravity pairs on a simulation of a free-flying supersonic aircraft in straight and level flight. This study examines nine routes first used in References 4 and 5 to study the impact of world-gravity pairs on a free-flying subsonic aircraft: a north route starting at the equator, a south route starting near $80^{\circ}$ latitude, a west route along the equator, and a west route near $35^{\circ}$ latitude, an east route near $80^{\circ}$ latitude, a north-east route starting at the equator, a north-west route starting at the equator, a $20^{\circ}$ track route starting at the equator, and a $340^{\circ}$ track route starting at the equator. For each route, the resulting trajectories of the world-gravity pairs were compared against the reference E/SE trajectory. Differences were analyzed as contributions from residual tangent gravity (isolated by the E/PM and E/C pairs), Coriolis acceleration (isolated by the ENR/FA and $\mathrm{ENR} / \mathrm{C}$ pairs), and reduced surface fidelity (isolated by the S/SE pair).

The subsonic study of Reference 5 made the following recommendations based on its results:

- Use the S/SE world gravity pair when reducing the world model to a sphere

- Use the ENR/FA (or ENR/C) world gravity pair(s) when reducing gravity fidelity

- SNR/FA and SNR/C world gravity pairs perform nearly as well as ENR/FA and ENR/C, allowing the user to reduce fidelity of both the world model and the sphere.

- Use of E/PM, E/C, S/PM, and S/C were not recommended due to their inconsistent performance relative to the non-rotating world gravity pairs.

One obvious difference in the supersonic study is that the non-rotating world pairs generally hold the worst performance. Only at the completion of the North-East from Equator and $20^{\circ}$ Track from Equator do the SNR/FA and $\mathrm{SNR} / \mathrm{C}$ routes out-perform all but the E/PM and E/C pairs. Only in the West from Equator route does the ENR/FA and ENR/C produce no error but that is true of all ellipsoid world-gravity pairs. The worsening performance of the non-rotating world pairs is an expected outcome from doubling the aircraft velocity, which also doubles the Coriolis acceleration that is absent from these world-gravity pairs. For the rotating world gravity pairs, errors at mid-route support different recommendations than errors at completion due to the growing error contribution from reduced surface fidelity. At mid-route, the S/SE trajectory has the lowest error for all routes with large north-south travel except for South from $80^{\circ}$ Latitude where it is within $5 \mathrm{nmi}$ of the lower error S/PM and S/C pairs. In the routes that primarily travel East-West, S/SE has the lowest error only in the West from $35^{\circ}$ Latitude route. In the West from Equator and East form $80^{\circ}$ latitude routes, it is out-performed by the E/PM and E/C pairs. In all cases, the S/SE trajectory error is less than $21 \mathrm{nmi}$. The E/PM and E/C pairs consistently have the second lowest error except from 
the West from Equator and East from $80^{\circ}$ Latitude routes where these pairs have the lowest error. The E/PM and $\mathrm{E} / \mathrm{C}$ trajectories have errors of less than $25 \mathrm{nmi}$ except for the West from $35^{\circ}$ Latitude route where its error is 33 $\mathrm{nmi}$. Except as noted above, the S/PM and S/C trajectories are in third place and have errors between $\sim 30 \mathrm{nmi}$ and $42.3 \mathrm{nmi}$ in five of the nine routes; those five include all four routes with tracks other than $0^{\circ} / 180^{\circ}$ or $90^{\circ} / 270^{\circ}$. Therefore, for simulations traveling less than $4800 \mathrm{nmi}$, the results support the following recommendations:

- If reducing the fidelity of the world model, use S/SE.

- If reducing the fidelity of the gravity model, use E/PM or E/C.

- If reducing both world and gravity fidelity is required, S/PM and S/C are the only viable options. However, these two pairs do generate elevated errors on the routes with tracks other than $0^{\circ} / 180^{\circ}$ or $90^{\circ} / 270^{\circ}$.

- ENR/FA, ENR/C, SNR/FA, and SNR/C are not recommended.

For full length of travel in the scenario ( 9600 nmi), the S/SE pair performs well only in routes with a $0^{\circ} / 180^{\circ}$ track or on routes with a $90^{\circ} / 270^{\circ}$ track at low to mid latitudes. E/PM and E/C are the only world-gravity pairs whose error does not exceed $\sim 36 \mathrm{nmi}$ in any scenario. At completion, the E/PM and E/C pairs generate errors of less than $30 \mathrm{nmi}$ under all routes except the West from $35^{\circ}$ Latitude route and best the rotating sphere pairs in six of the nine scenarios. The S/PM and S/C trajectories show wide variation in performance at completion. The trajectories produce errors less than $30 \mathrm{nmi}$ in five scenarios but produce errors of more than 67 nmi in the other four. Therefore, for simulations traveling more than $4800 \mathrm{nmi}$, the results support the following recommendations.

- For general use, only reducing gravity fidelity is recommended. Use the E/PM or E/C pair.

- If reducing fidelity of the world model is required, the simulation should stick with tracks near $0^{\circ} / 180^{\circ}$ or $90^{\circ} / 270^{\circ}$ and use the S/PM or S/C world gravity pair. S/SE also performs well on tracks of $0^{\circ} / 180^{\circ}$ or $90^{\circ} / 270^{\circ}$ but only at low to mid latitudes. The rotating sphere world-gravity pairs may generate large errors on other tracks and are not recommended for this use.

- ENR/FA, ENR/C, SNR/FA, and SNR/C are not recommended.

There is little in this study to recommend the point-mass gravitation model over the constant gravitation model. Both models perform identically except for the two north-south routes where the constant gravitation model provides about a 1.2 to $2.3 \mathrm{nmi}$ improvement in maximum error. However, this may be an artifact of flying straight and level flights were the vehicle is trimmed to counteract the initial gravity. Greater differences may emerge with routes containing altitude changes. Gravity model choice may also have a larger impact on high altitude aircraft simulations. Another area for further investigation is the effect that options for lateral trimming of the aircraft have on results. In the subsonic investigation, the lateral control surfaces were left in the neutral position. But, in this study, it became necessary to cancel initial yaw and roll acceleration using the lateral control surfaces in order to extend error trends from the subsonic study to the results of the supersonic study in some scenarios. The difference in relative performance of the world gravity pairs at the mid-point and at completion also invite the question of whether the subsonic aircraft would see similar trends if its flight were extended to 9600 nmi. Finally, because curvature differences in both lines of latitude and longitude appear to accelerate the error growth of the S/SE trajectory in the last half of scenarios with north and east travel, the question arises whether this same error growth would be seen with other choices for the Earth's radius. 


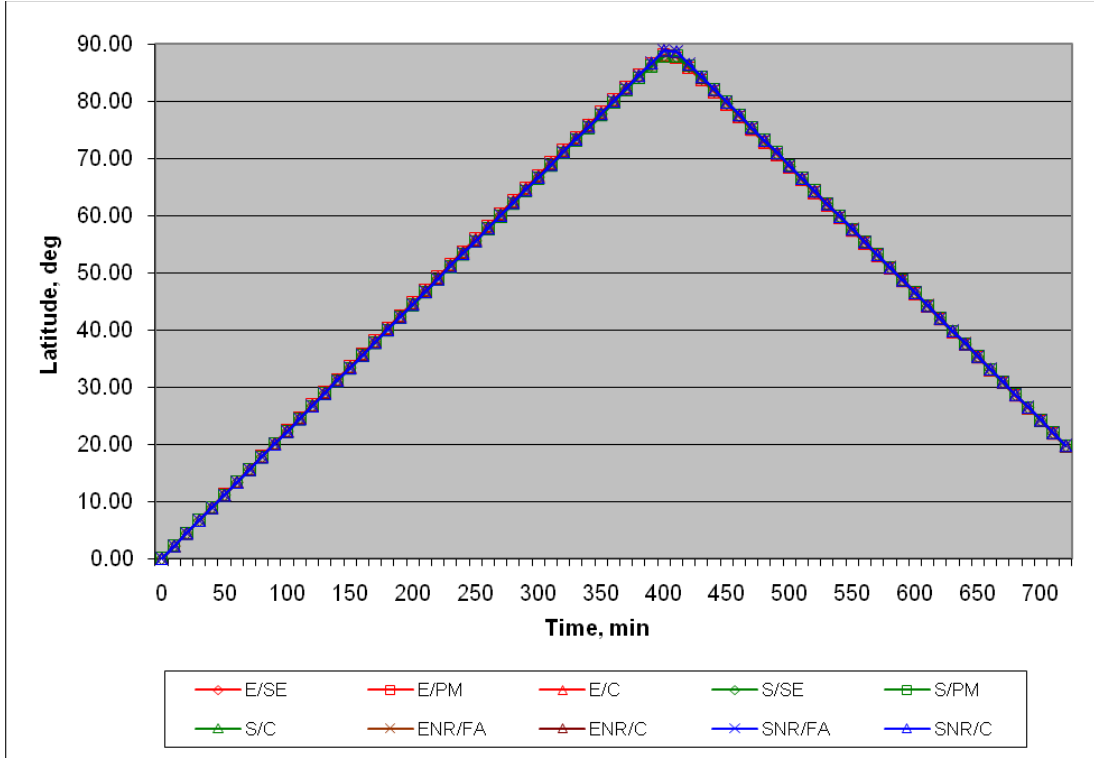

Figure 1 North from Equator - Latitude vs. Time

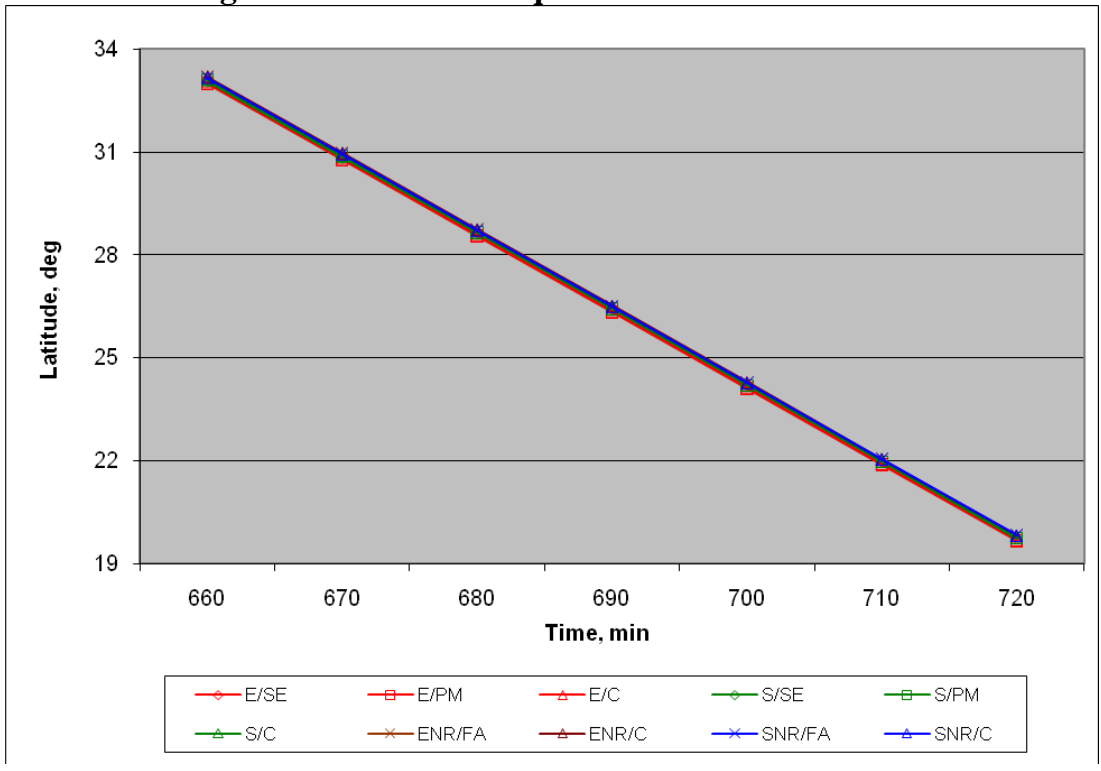

Figure 3 North from Equator - Latitude vs. Time (Last Hour)

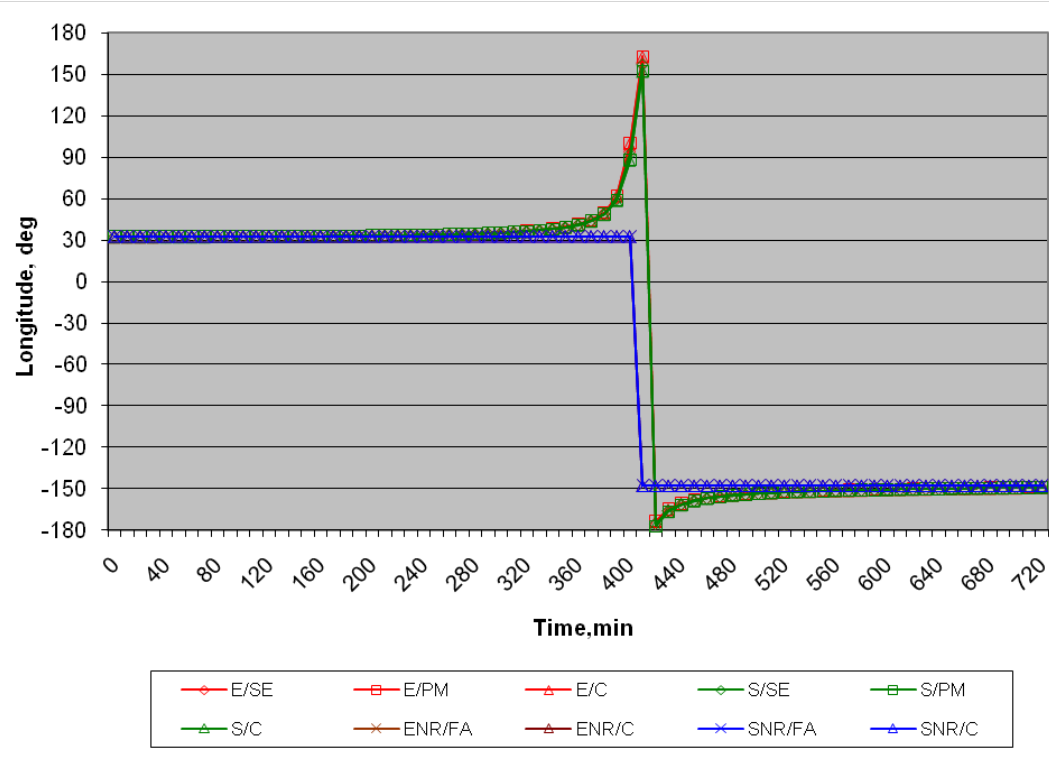

Figure 2 North from Equator - Longitude vs. Time

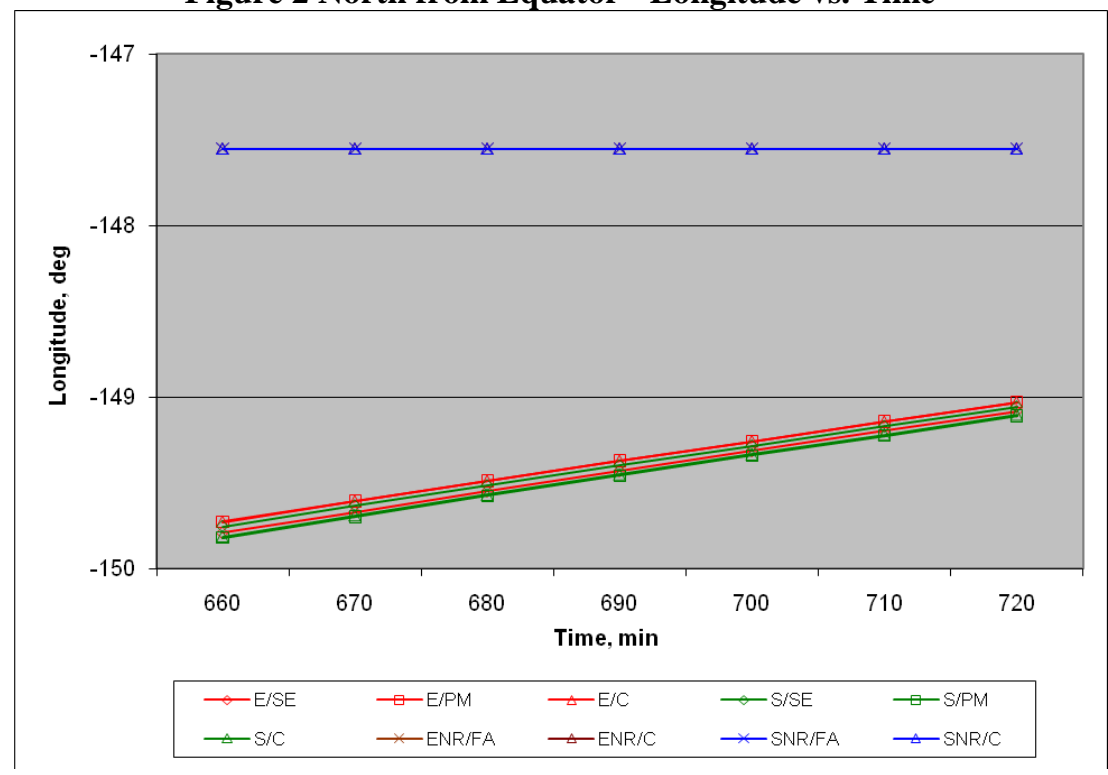

Figure 4 North from Equator - Longitude vs. Time (Last Hour) 


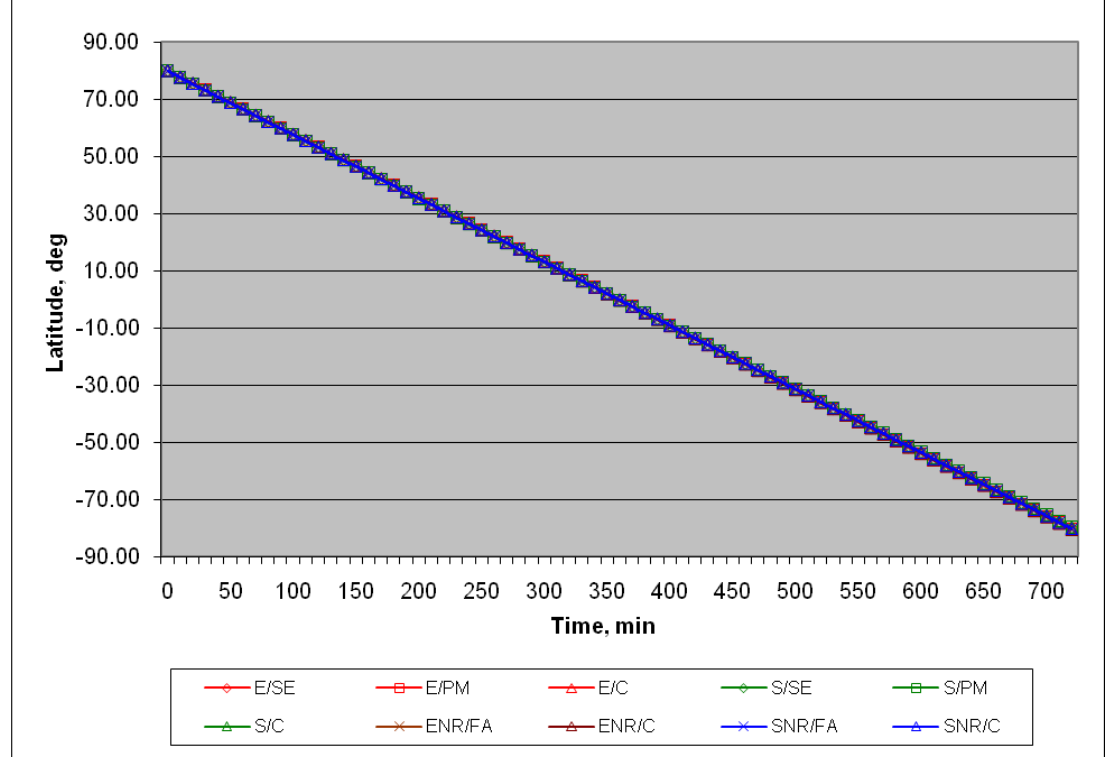

Figure 5 South from $80^{\circ}$ Latitude - Latitude vs. Time

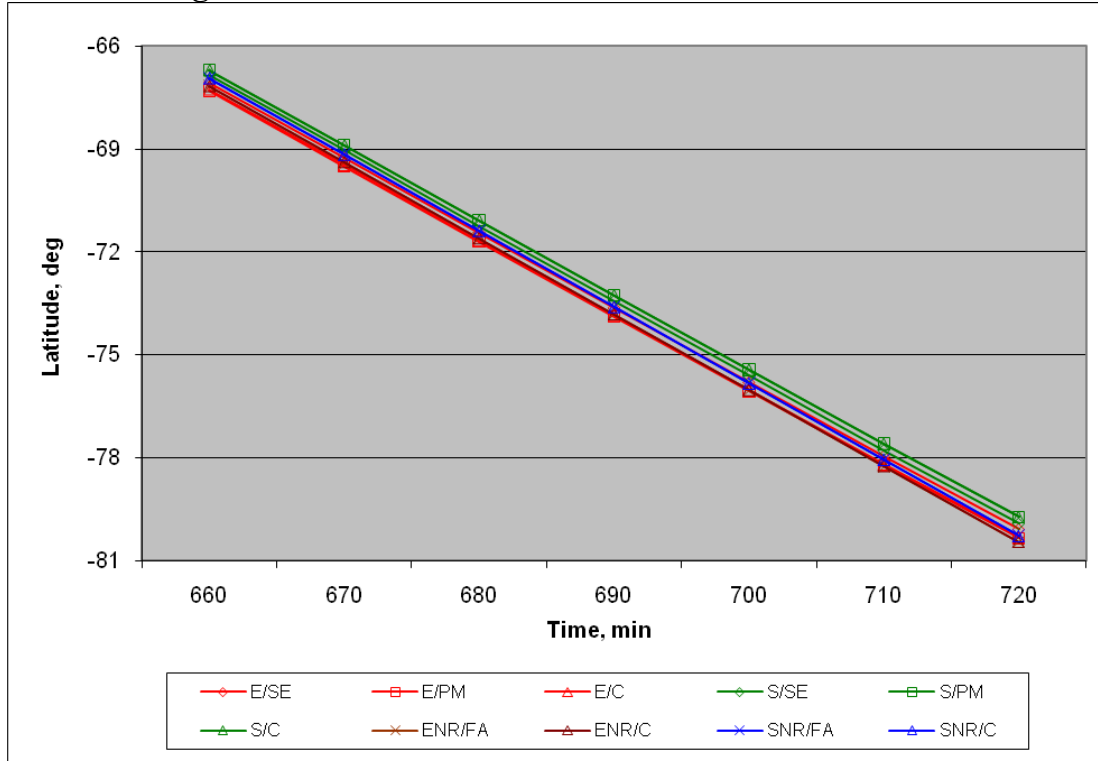

Figure 7 South from $80^{\circ}$ Latitude - Latitude vs. Time (Last Hour)

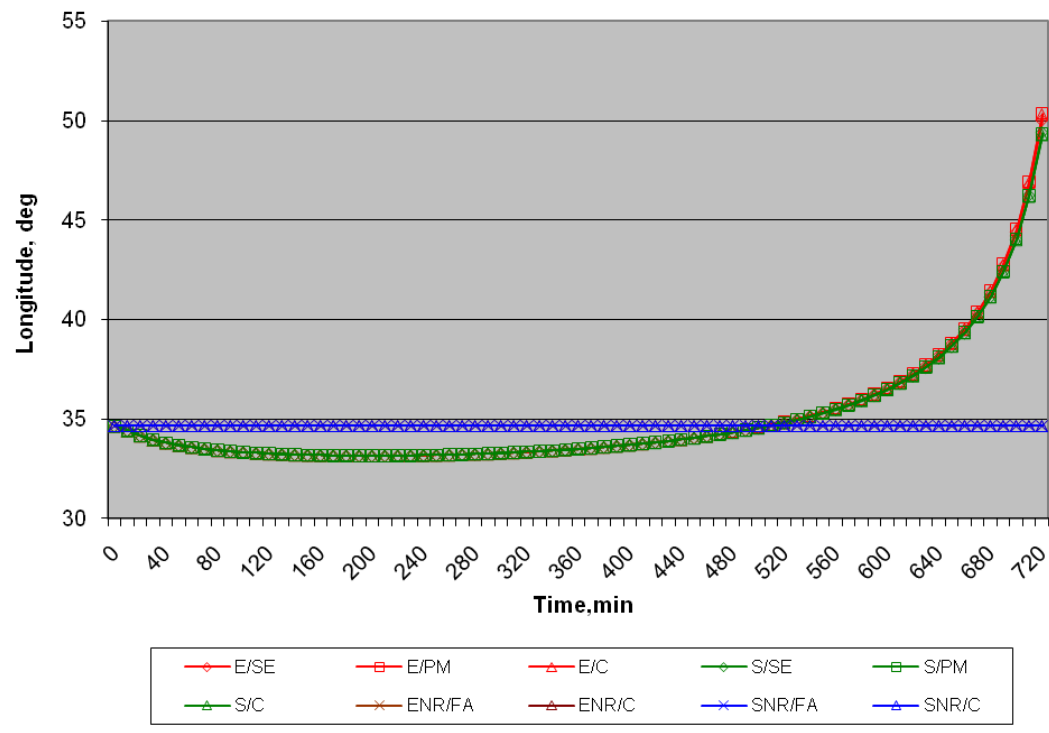

Figure 6 South from $80^{\circ}$ Latitude - Longitude vs. Time

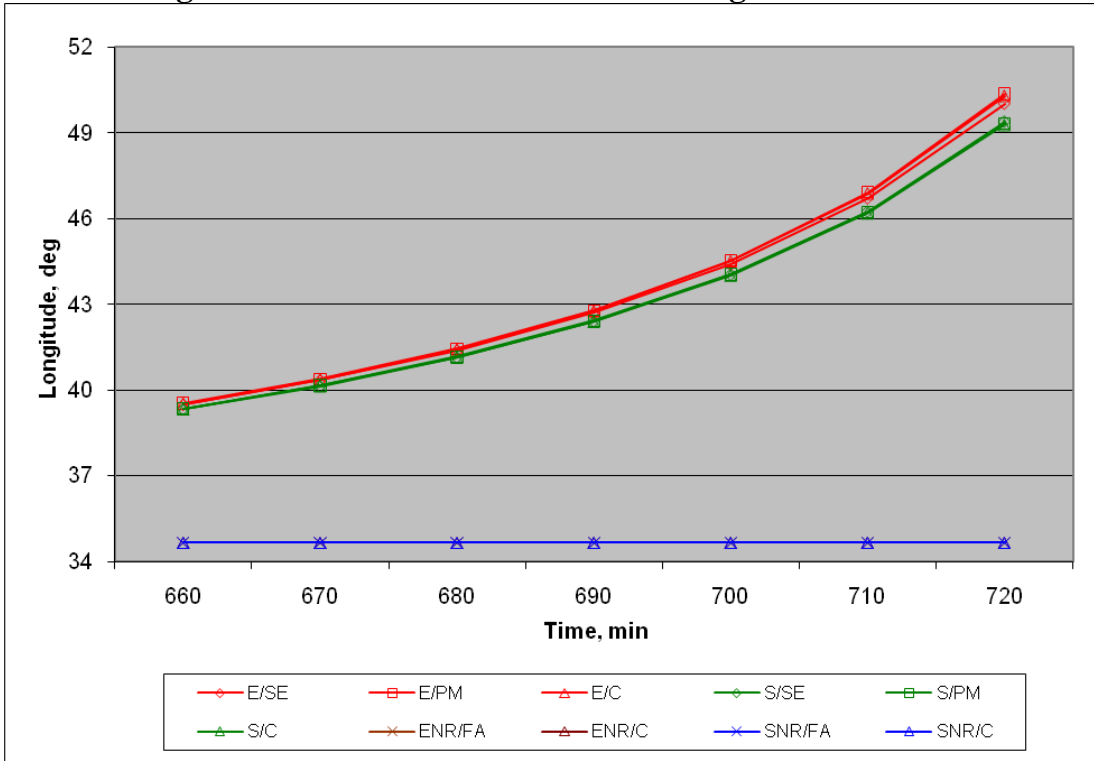

Figure 8 South from $80^{\circ}$ Latitude - Longitude vs. Time (Last Hour) 

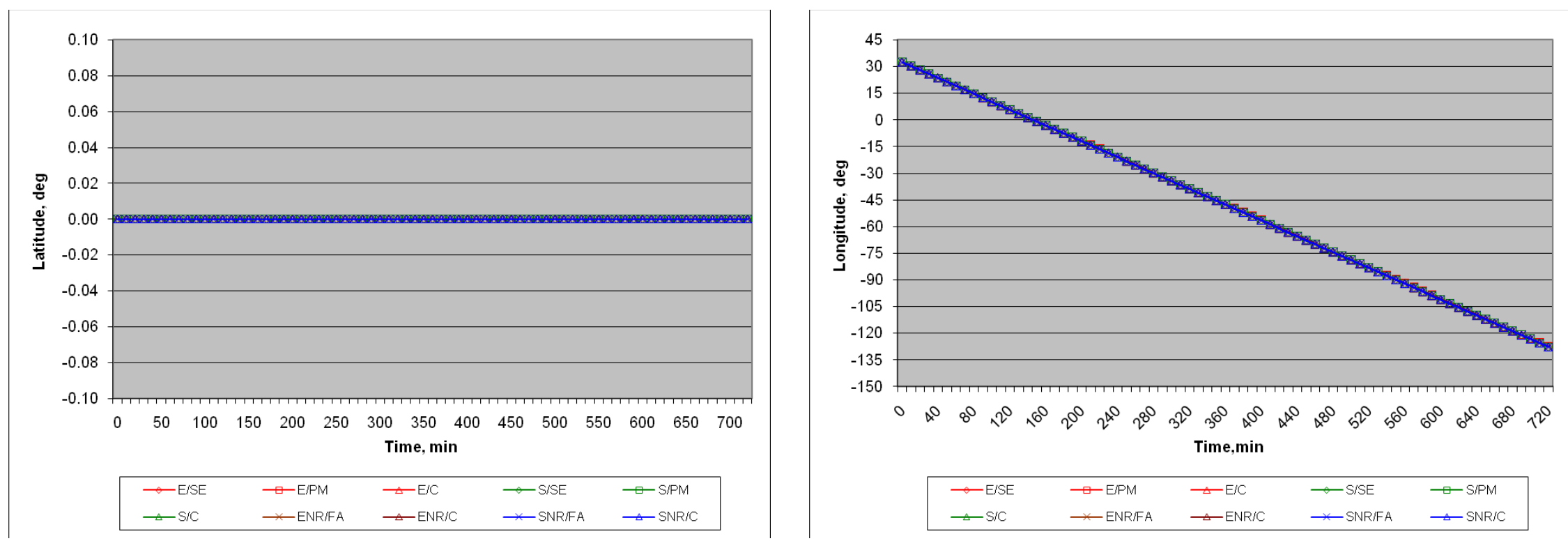

Figure 9 West from Equator - Latitude vs. Time

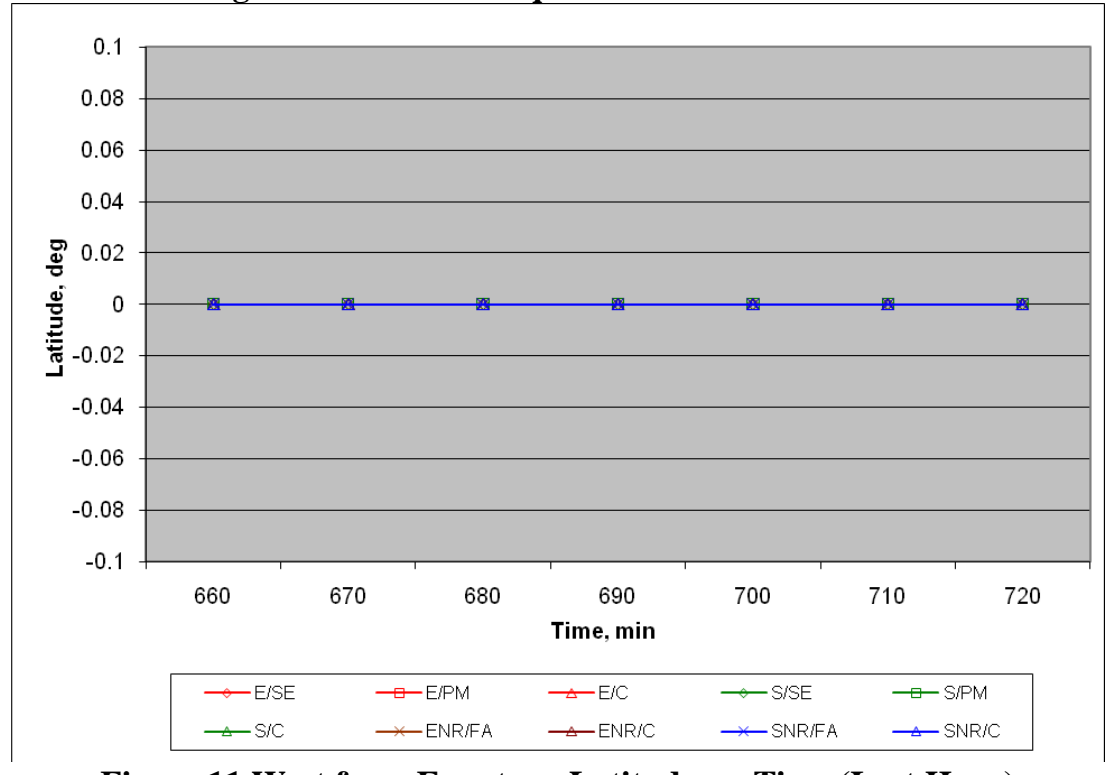

Figure 11 West from Equator - Latitude vs. Time (Last Hour)

Figure 10 West from Equator - Longitude vs. Time

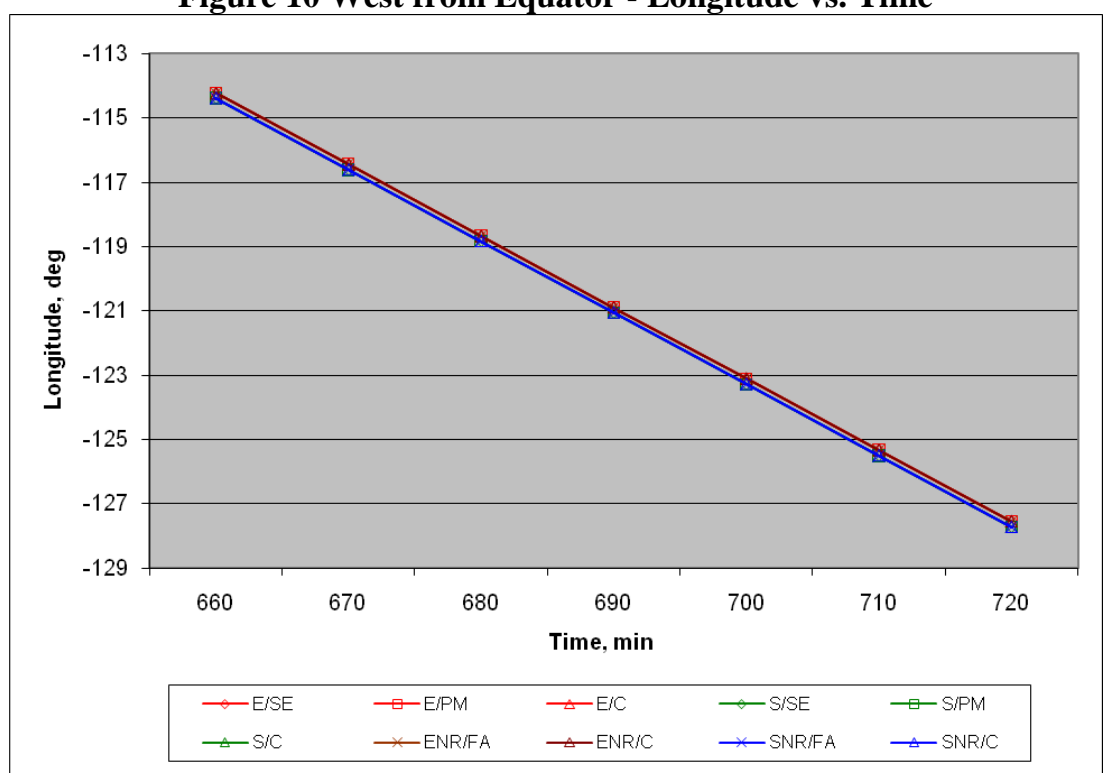

Figure 12 West from Equator - Longitude vs. Time (Last Hour) 


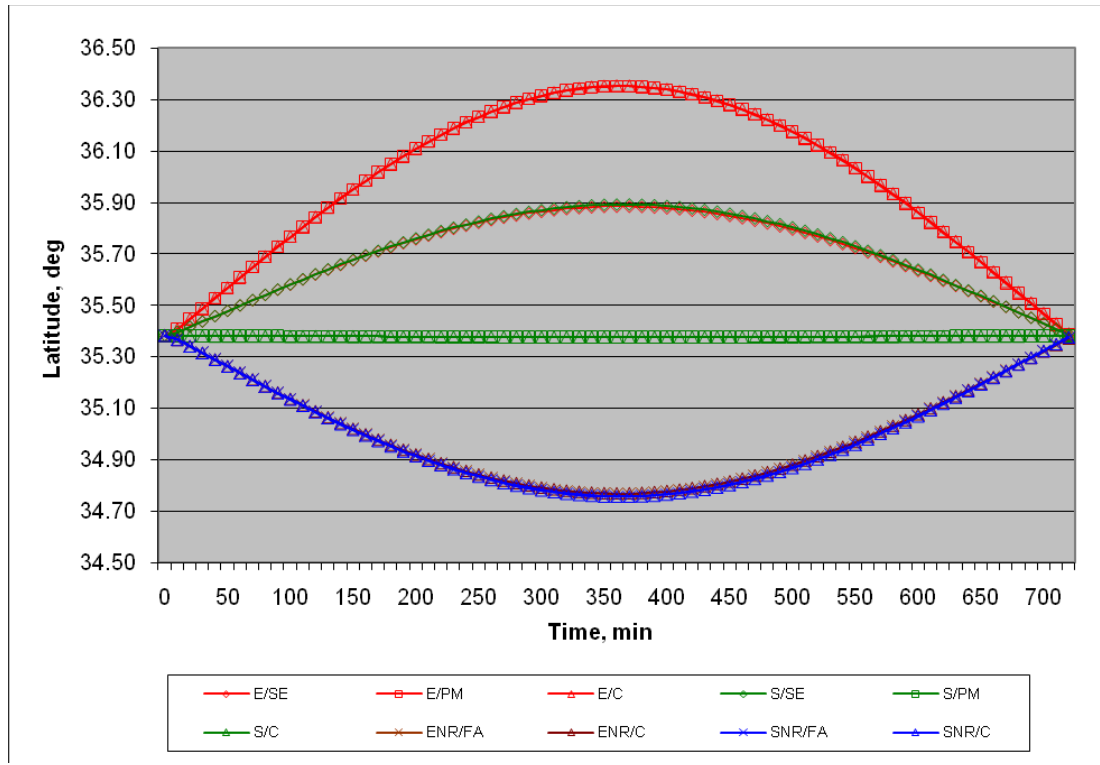

Figure 13 West from $35^{\circ}$ Latitude - Latitude vs. Time

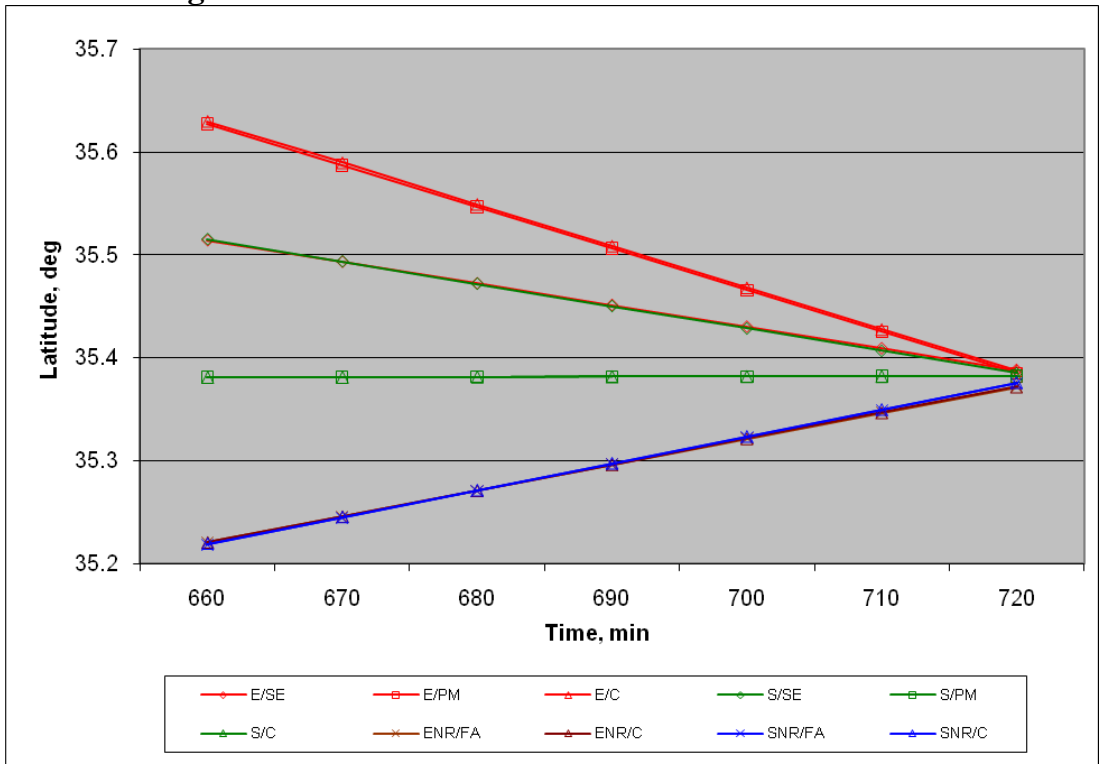

Figure 15 West from $35^{\circ}$ Latitude - Latitude vs. Time (last hour)

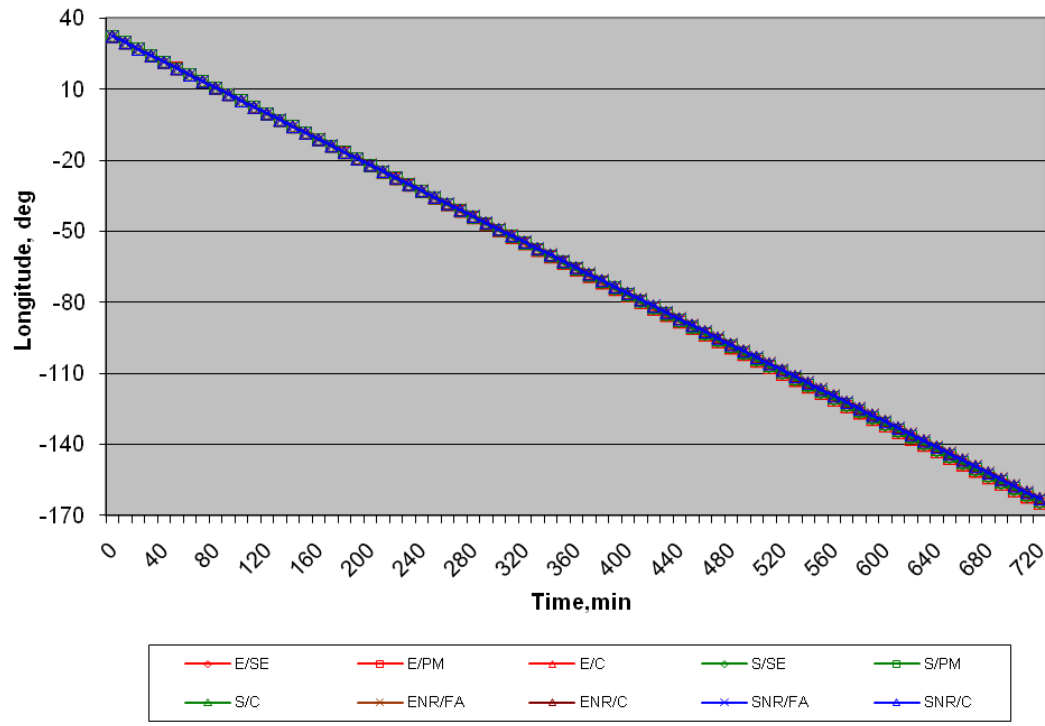

Figure 14 West from $35^{\circ}$ Latitude - Longitude vs. Time

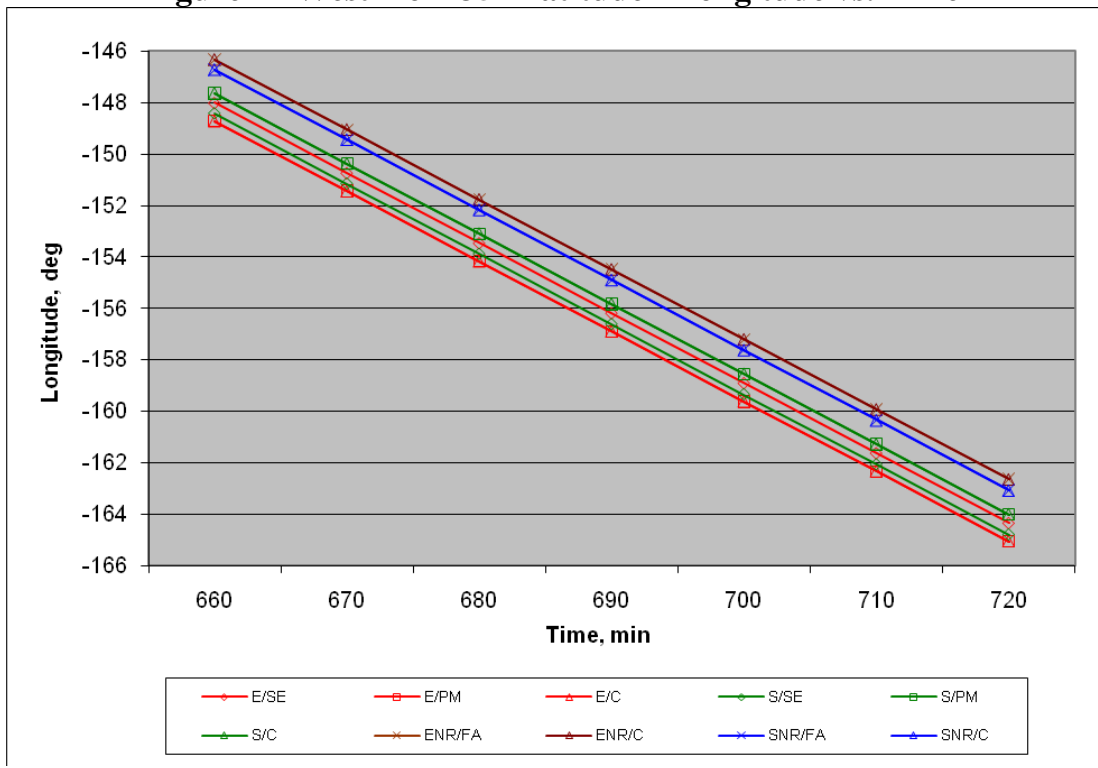

Figure 16 West from $35^{\circ}$ Latitude - Longitude vs. Time (Last Hour) 


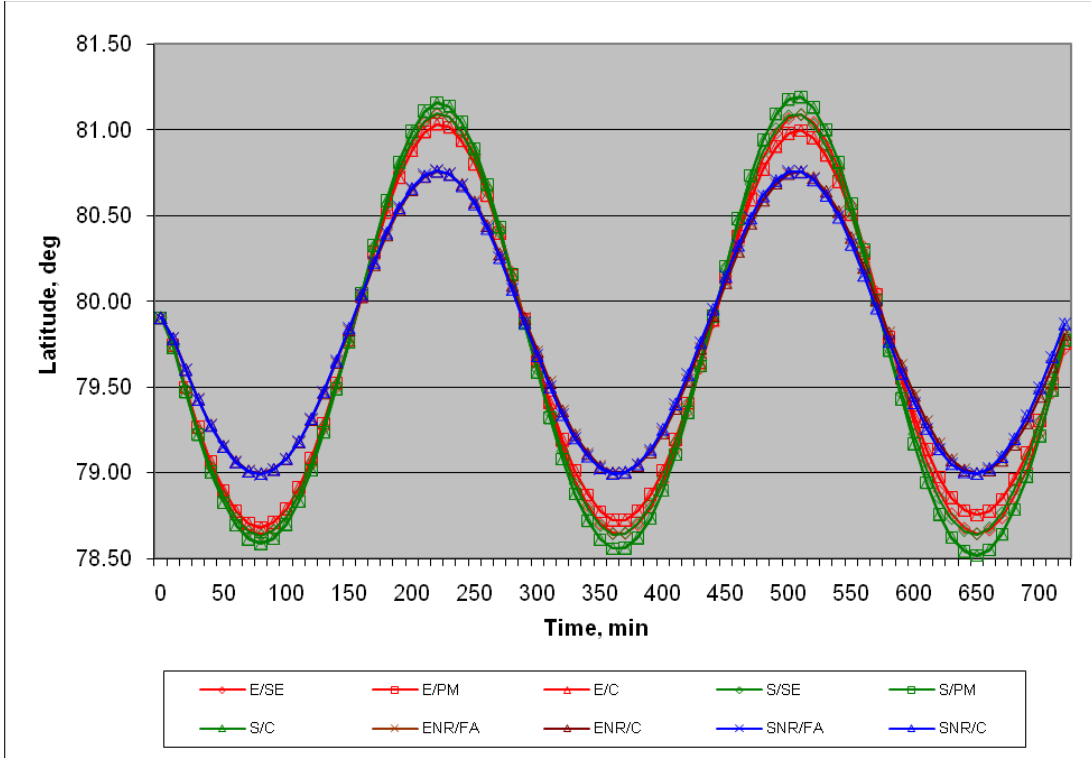

Figure 17 East from $80^{\circ}$ Latitude - Latitude vs. Time

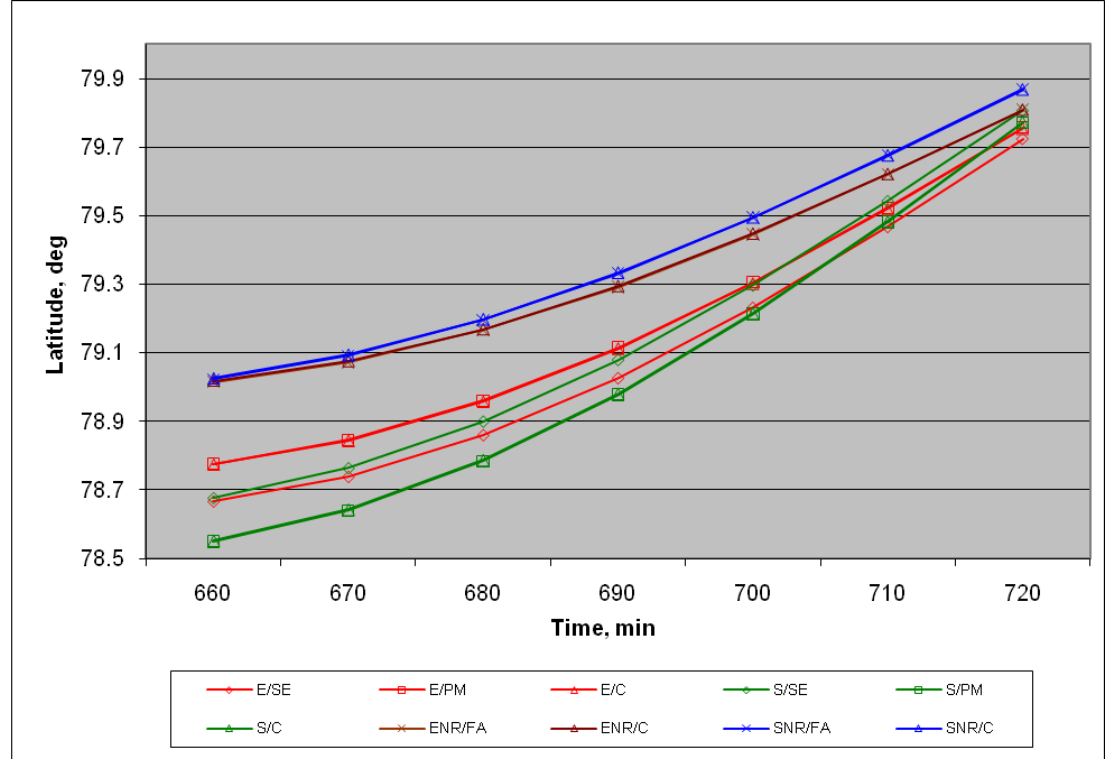

Figure 19 East from $80^{\circ}$ Latitude - Latitude vs. Time (Last Hour)

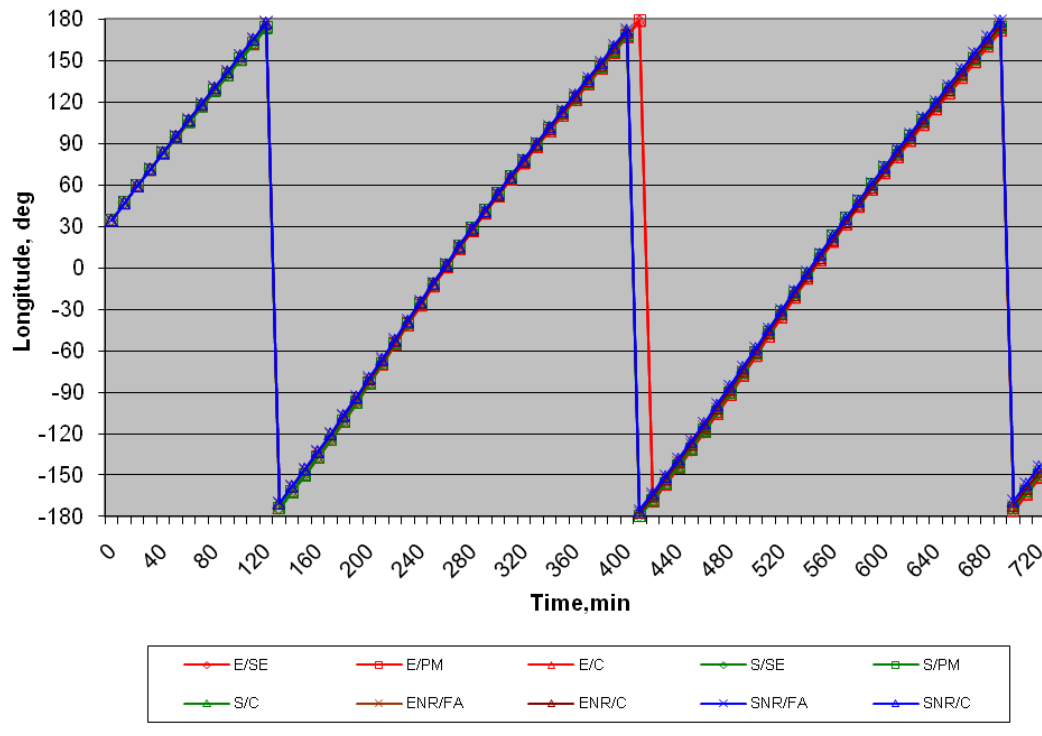

Figure 18 East from $80^{\circ}$ Latitude - Longitude vs. Time

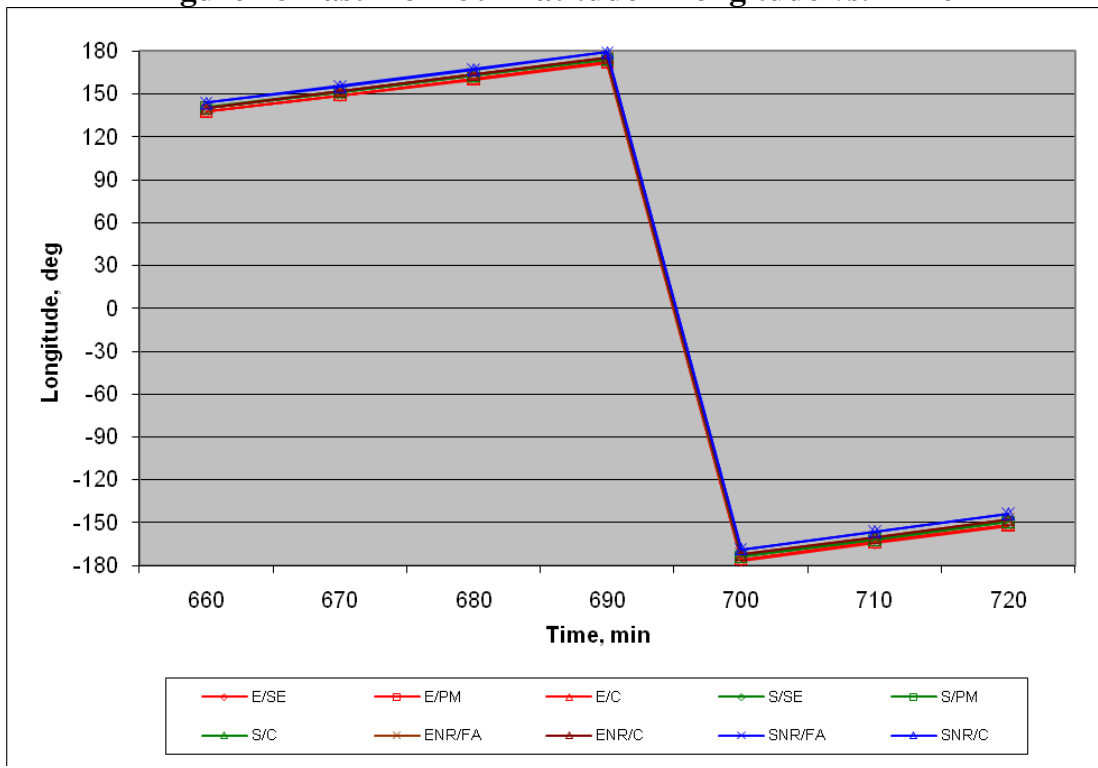

Figure 20 East from $80^{\circ}$ Latitude - Longitude vs. Time (Last Hour) 

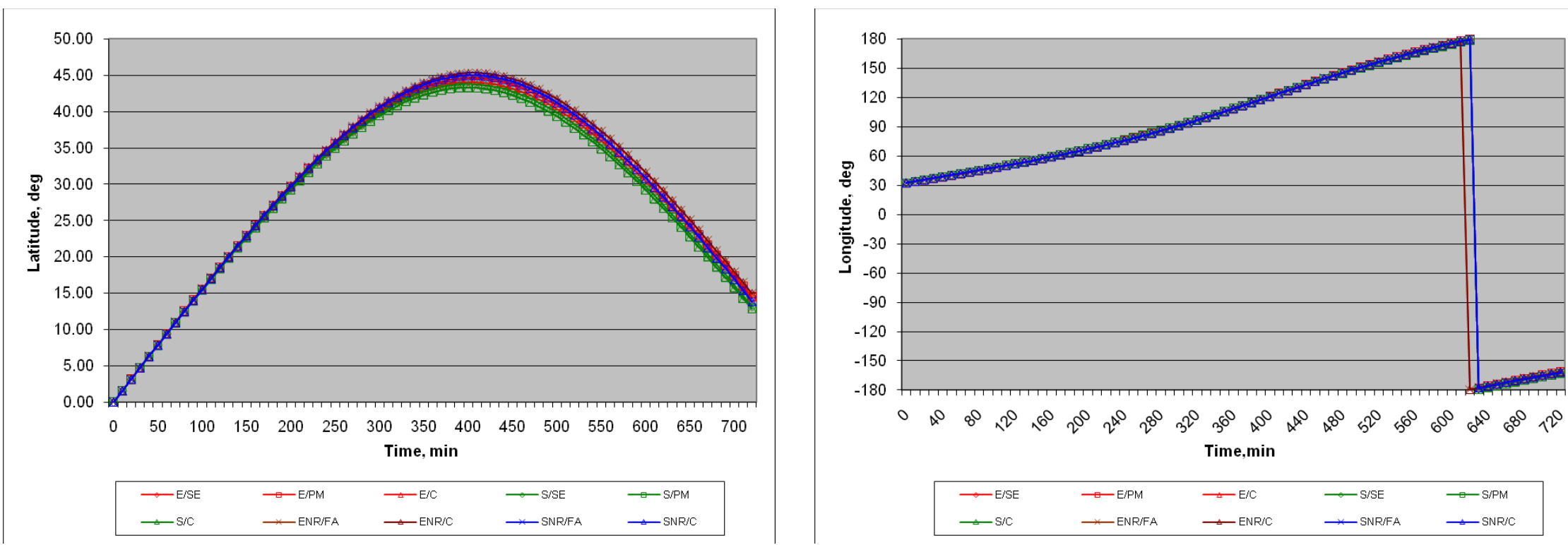

Figure 21 North-East from Equator - Latitude vs. Time

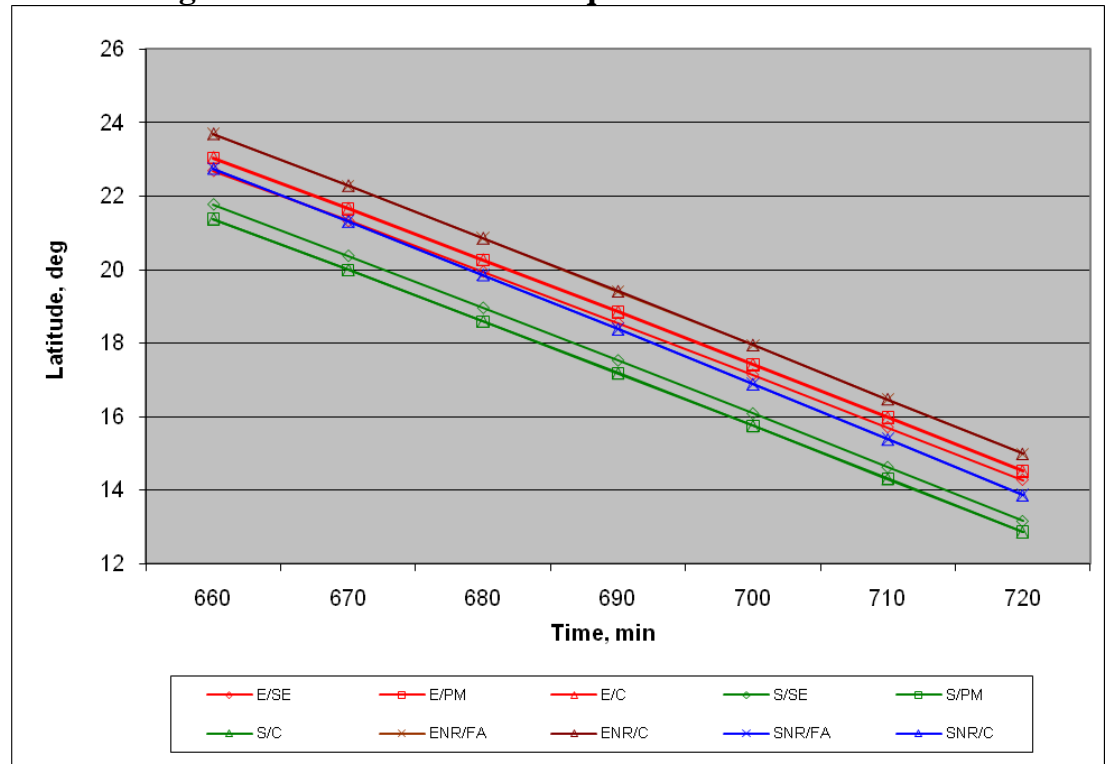

Figure 23 North-East from Equator - Latitude vs. Time (Last Hour)

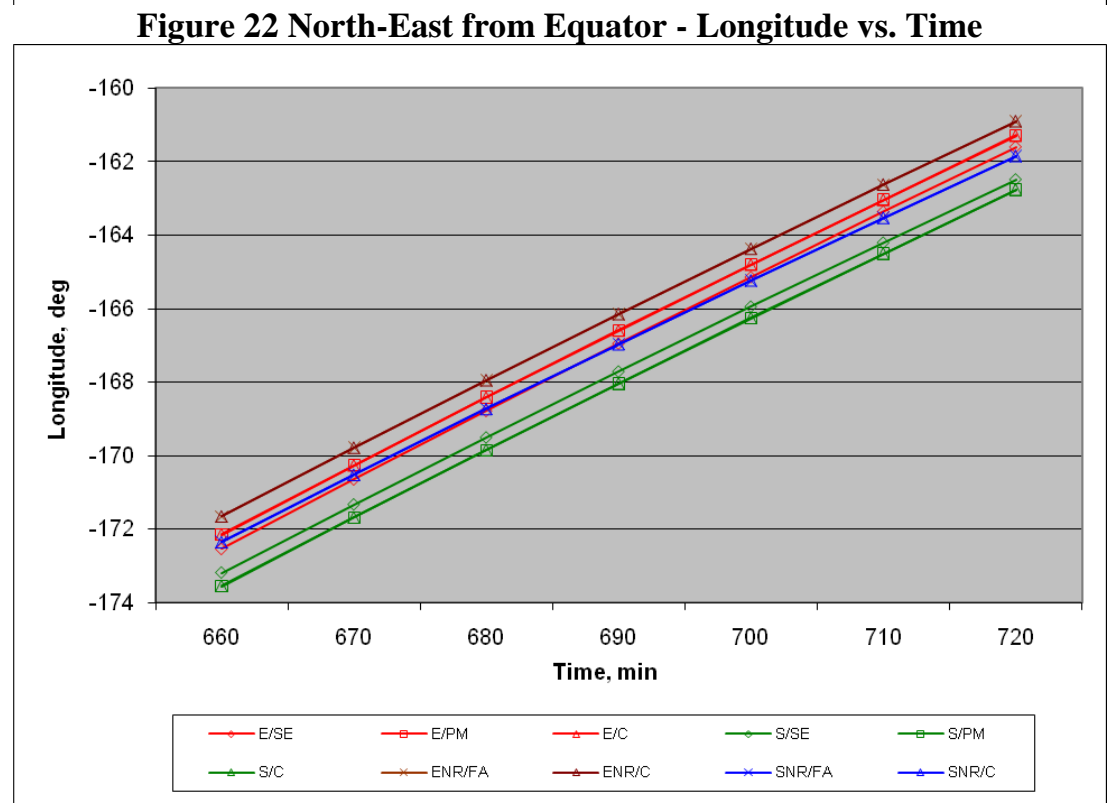

Figure 24 North-East from Equator - Longitude vs. Time (Last Hour) 


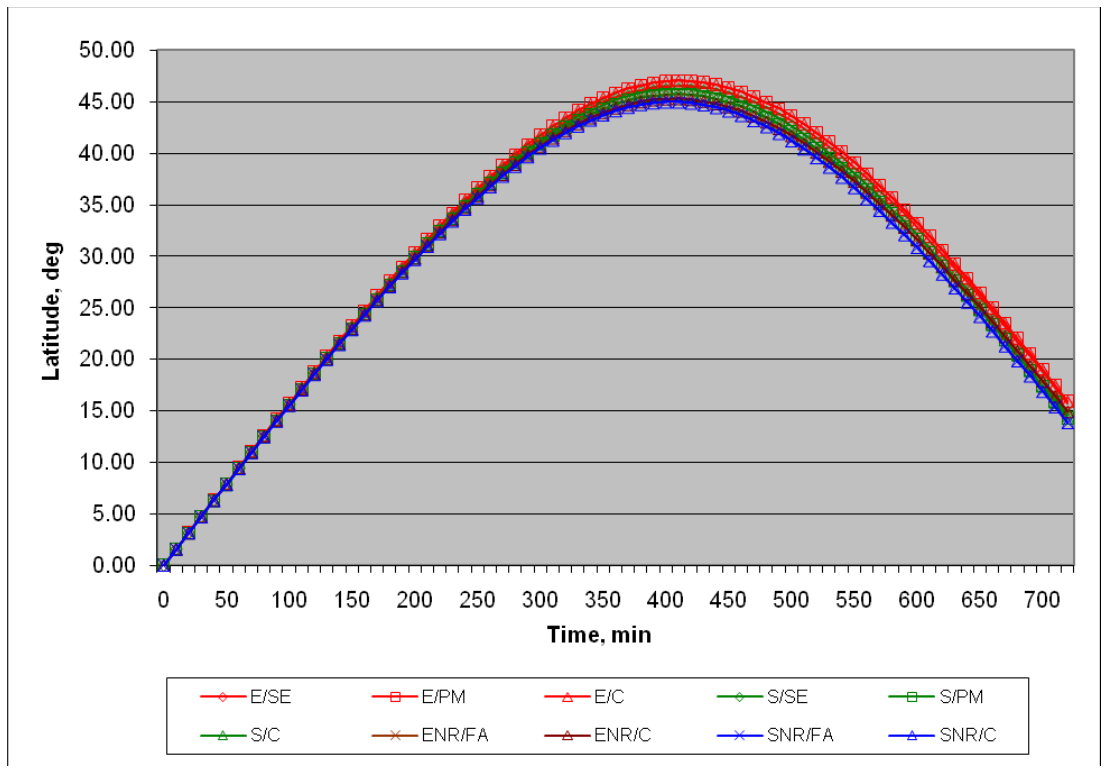

Figure 25 North-West from Equator - Latitude vs. Time

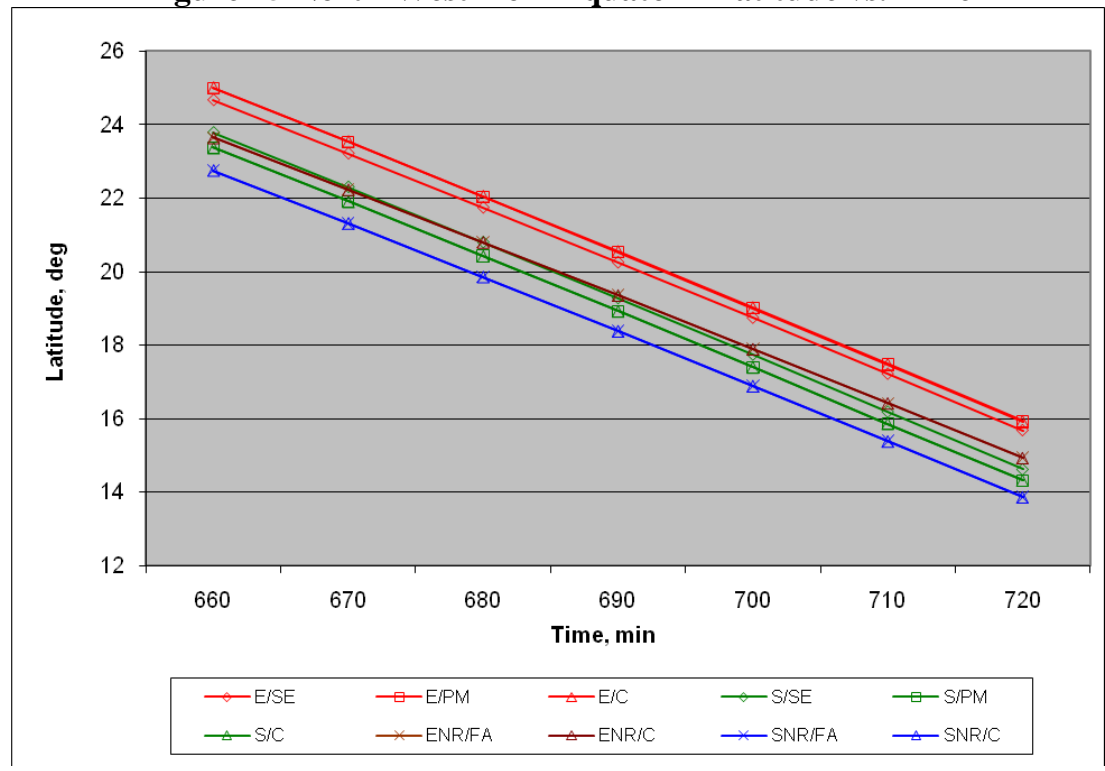

Figure 27 North-West from Equator - Latitude vs. Time (Last Hour)

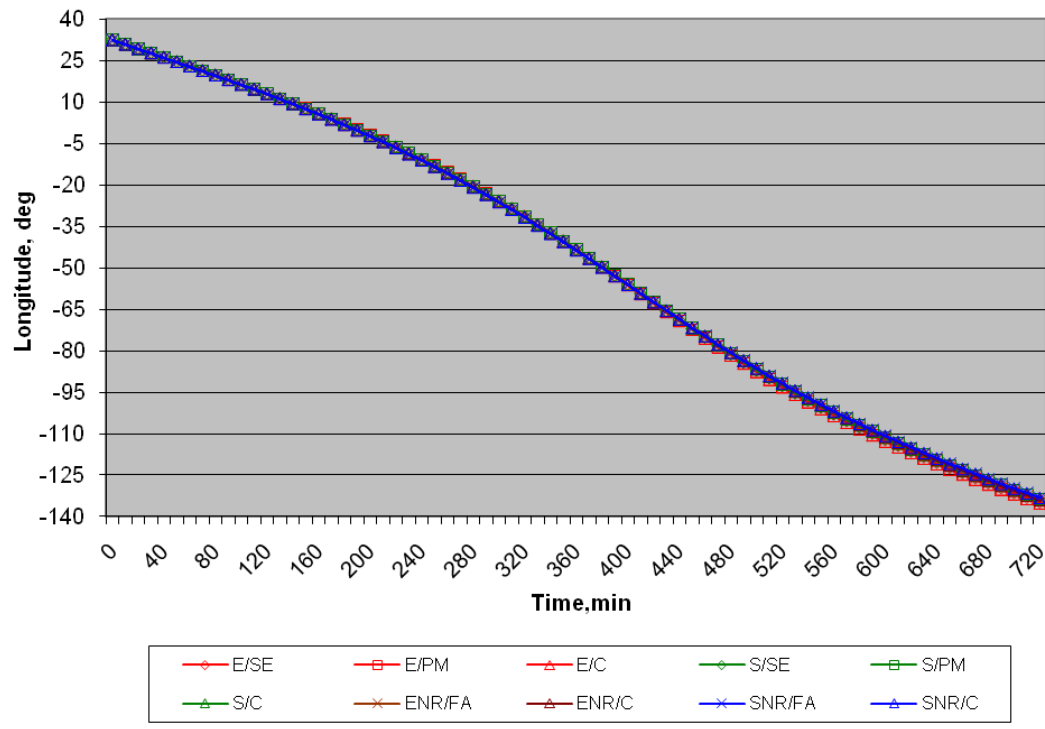

Figure 26 North-West from Equator - Latitude vs. Time

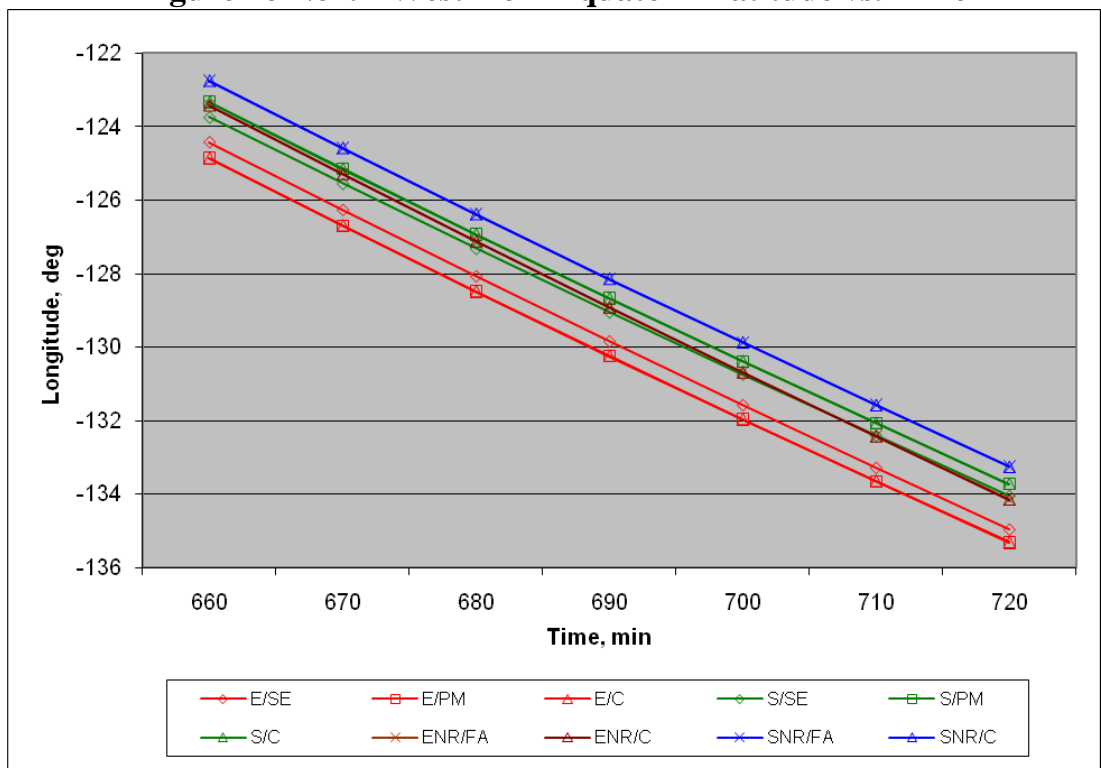

Figure 28 North-West from Equator - Longitude vs. Time (Last Hour) 


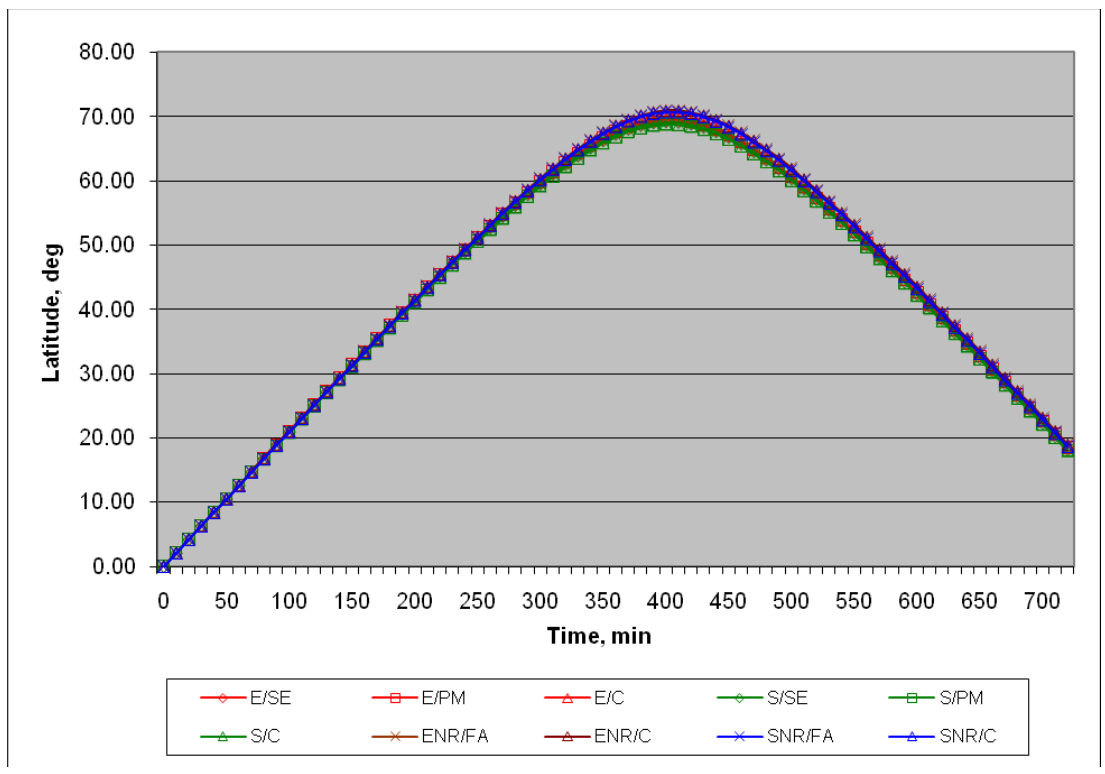

Figure $2920^{\circ}$ Track from Equator - Latitude vs. Time

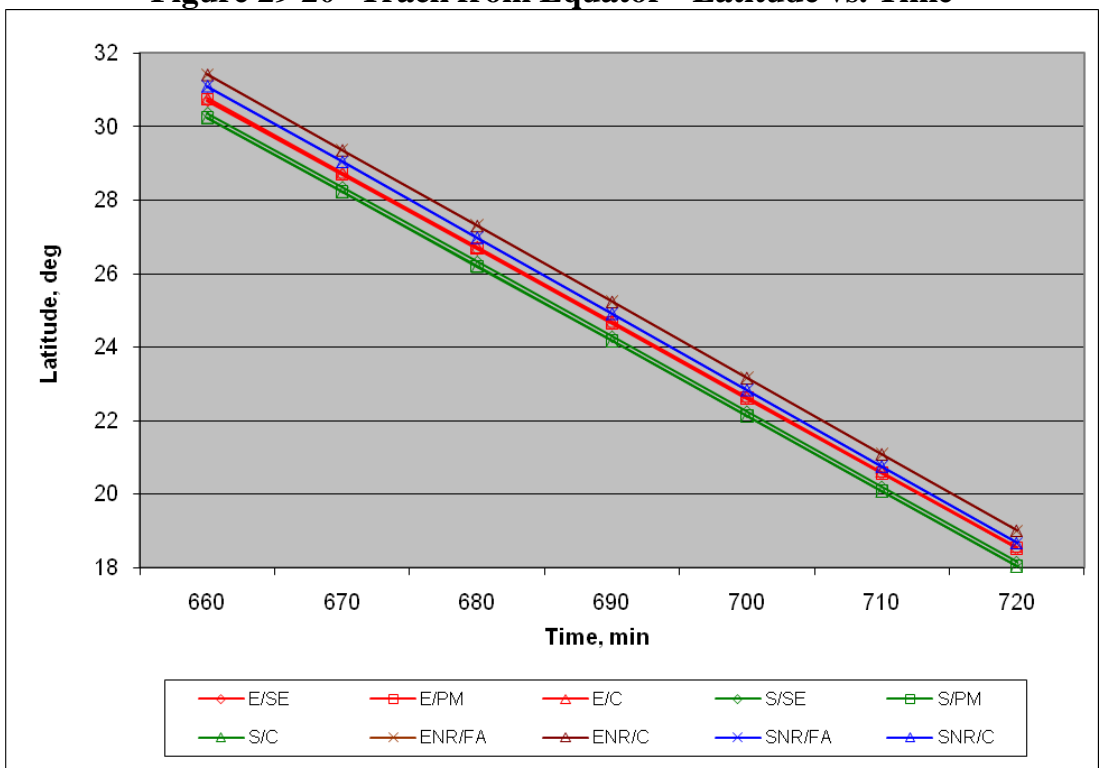

Figure $312^{\circ}$ Track from Equator - Latitude vs. Time (Last Hour)

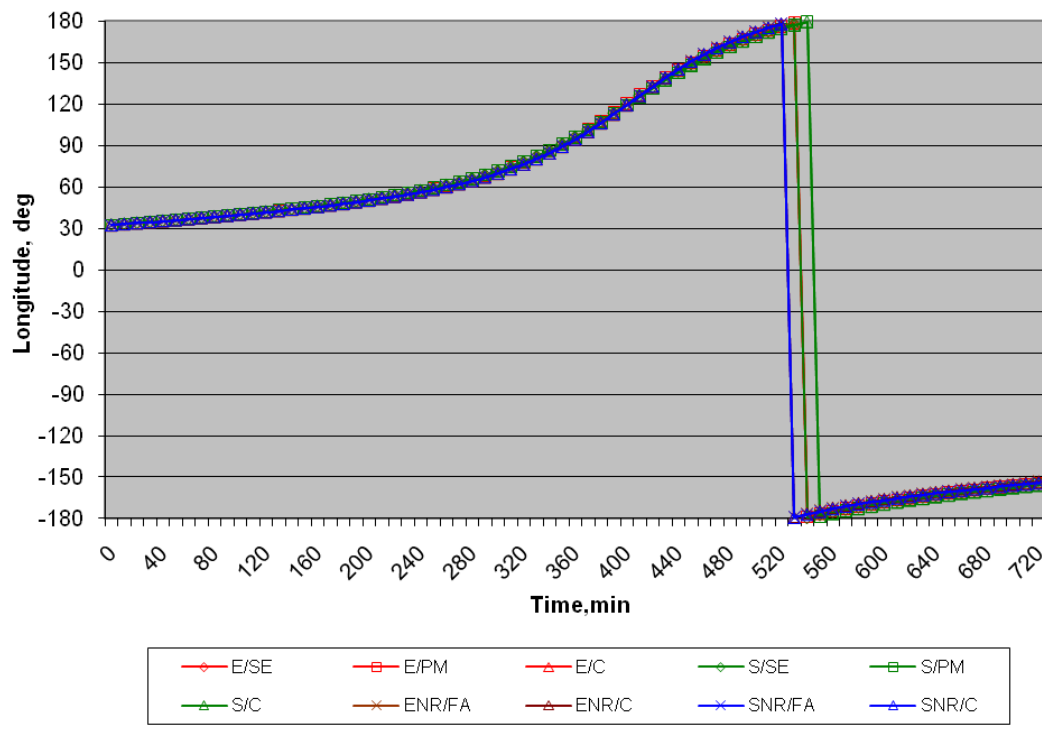

Figure $3020^{\circ}$ Track from Equator - Longitude vs. Time

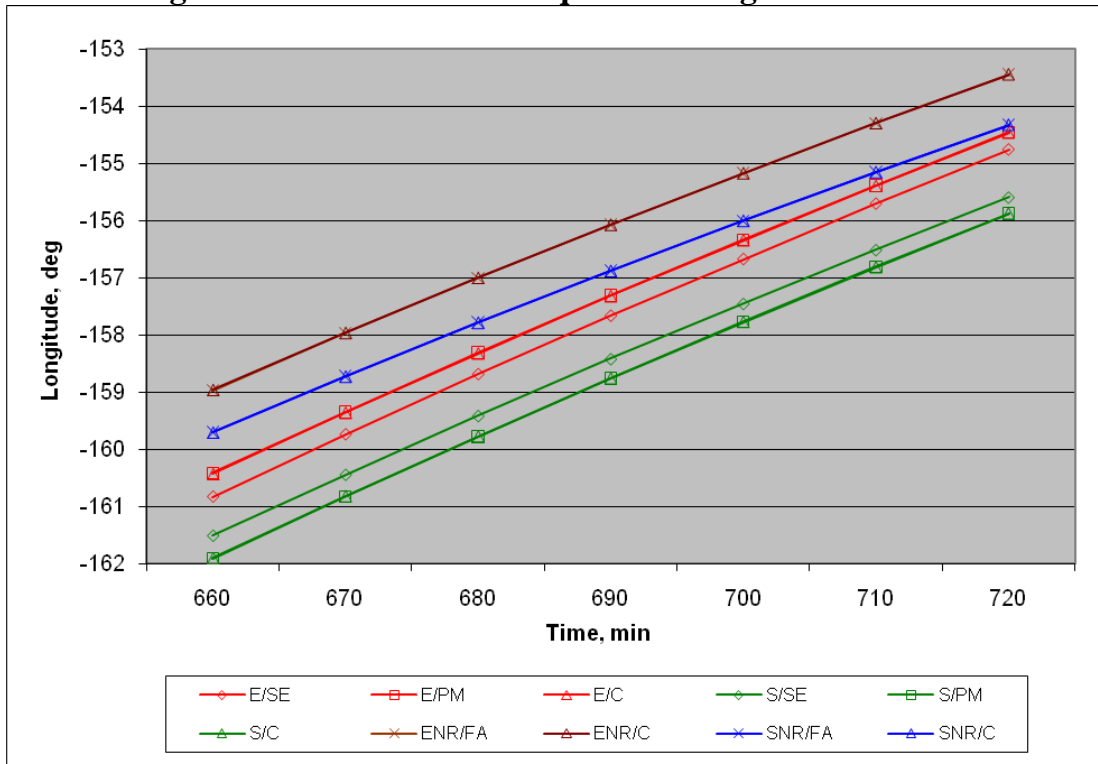

Figure $32 \mathbf{2 0}^{\circ}$ Track from Equator - Longitude vs. Time (Last Hour) 


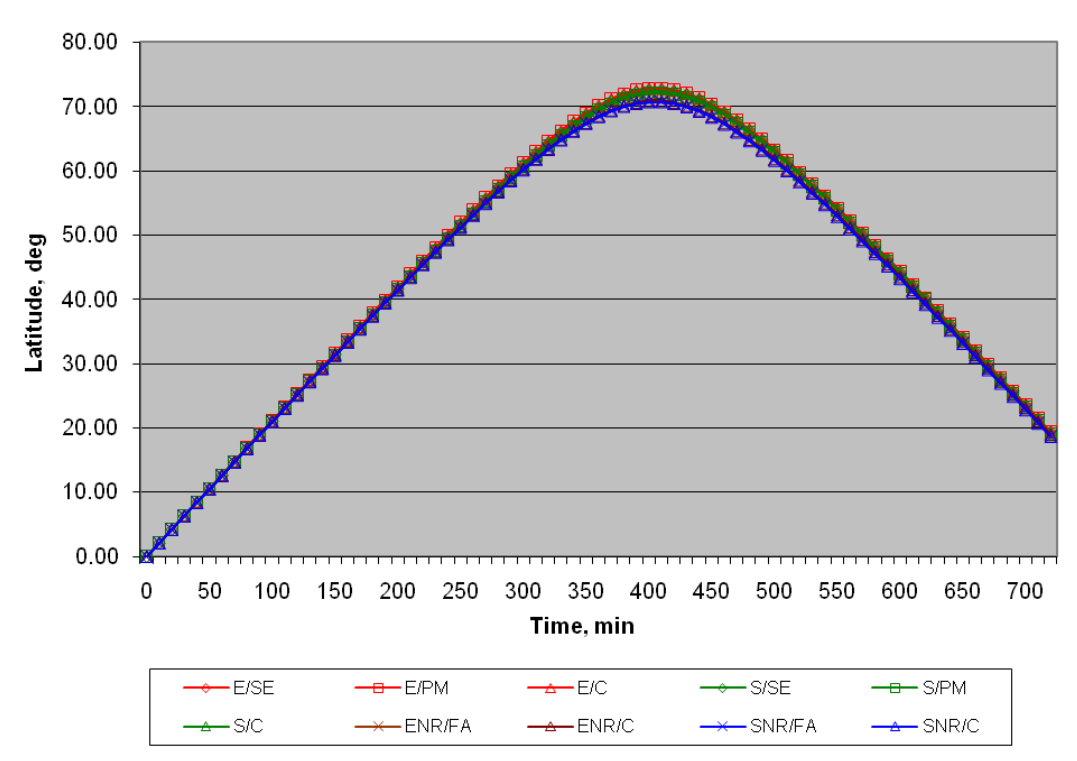

Figure $33340^{\circ}$ Track from Equator - Latitude vs. Time

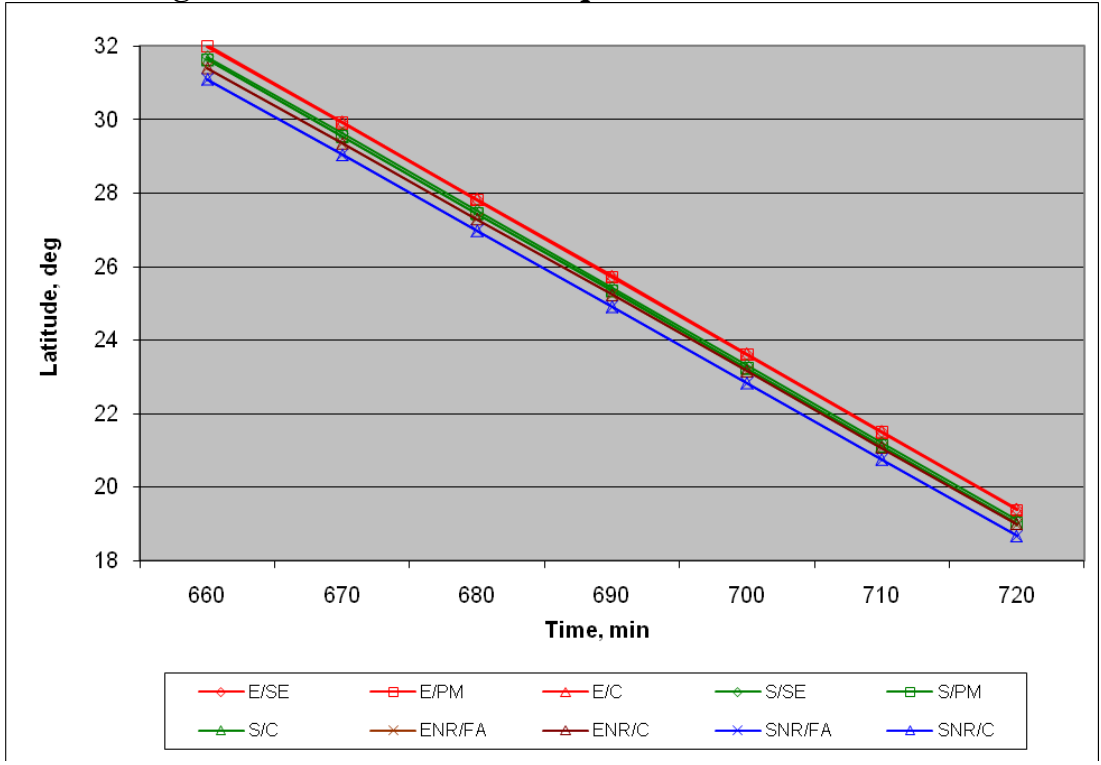

Figure $35340^{\circ}$ Track from Equator - Latitude vs. Time (Last Hour)

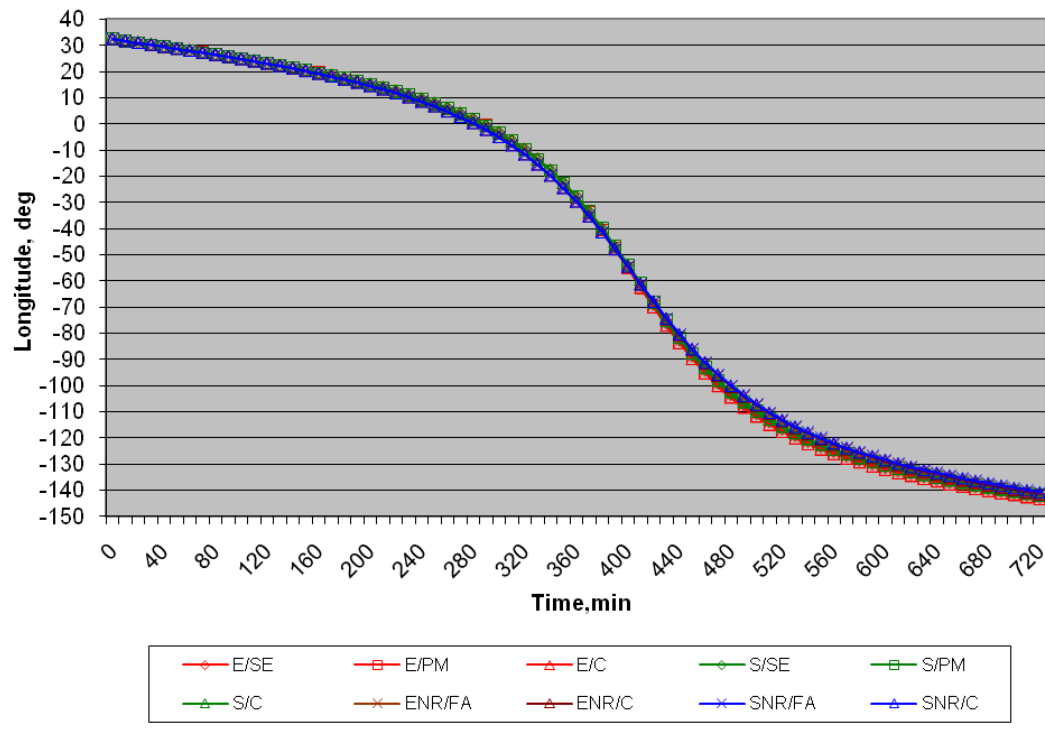

Figure $34340^{\circ}$ Track from Equator - Longitude vs. Time

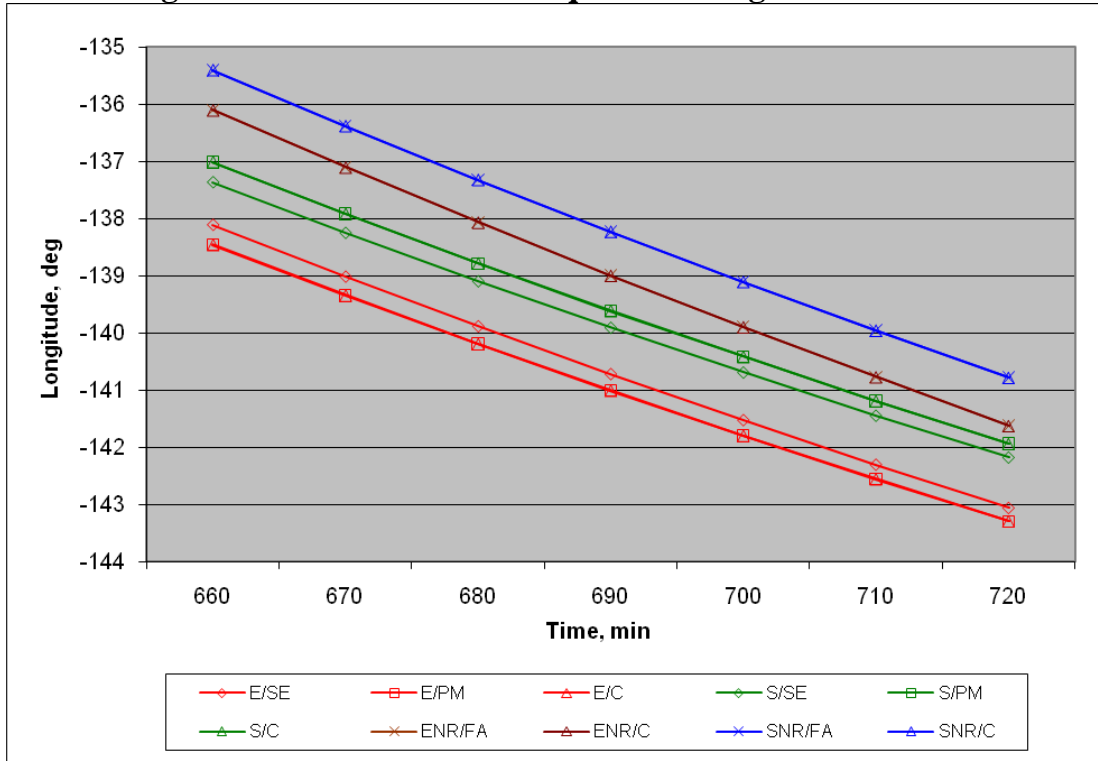

Figure $36340^{\circ}$ Track from Equator - Longitude vs. Time (Last Hour) 


\section{References}

1 R. Leslie, D. Geyer, K. Cunningham, M. Madden, P. Kenney, and P. Glaab, "LaSRS++: An Object-Oriented Framework for Real-Time Simulation of Aircraft,” AIAA Modeling and Simulation Technologies Conference and Exhibit, August 1998, Boston, Massachusetts, AIAA-98-4529.

2 Madden, Michael M. “Gravity Modeling for Variable Fidelity Environments”, AIAA-2006-6730, AIAA Modeling and Simulation Technologies Conference, Keystone, CO, August 21-24, 2006.

3 “Department of Defense World Geodetic System 1984", National Imagery and Mapping Agency, NIMA TR8350.2, Third Edition, Amendment 1, 3 January 2000.

4 Madden, Michael M. "Influence of World and Gravity Model Selection on Surface Interacting Vehicle Simulations", AIAA-2007-6370, AIAA Modeling and Simulation Technologies Conference, Hilton Head, SC, August 20-23, 2007.

5 Madden, Michael M. "Further Investigations of Gravity Modeling on Surface Interacting Vehicle Simulations”, AIAA2009-5700, AIAA Modeling and Simulation Technologies Conference, Chicago, IL, August 10-13, 2009.

6 Allen, William H. (Ed.), "Dictionary of Technical Terms for Aerospace Use”, National Aeronautics and Space Administration, Washington D.C., NASA SP-7, First Edition, 1965. Also available as an online database at URL: http://roland.lerc.nasa.gov/ dglover/dictionary/content.html [cited July 19, 2006].

7 Hofmann-Wellenhof, Bernhard and Moritz, Helmut, Physical Geodesy, SpringerWeinNewYork, New York, 2005, ISBN 3-211-23584-1. Sections Preface, 1.7, 2.1, 2.10.

8 Moritz, H. “Geodetic Reference System 1980”, Journal of Geodesy, Vol. 74, No.1, 2000, pp. 128-133. 